\title{
TOPOLOGY OF SUBVARIETIES OF COMPLEX SEMI-ABELIAN VARIETIES
}

\author{
YONGQIANG LIU, LAURENTIU MAXIM, AND BOTONG WANG
}

\begin{abstract}
We use the non-proper Morse theory of Palais-Smale to investigate the topology of smooth closed subvarieties of complex semi-abelian varieties, and that of their infinite cyclic covers. As main applications, we obtain the finite generation (except in the middle degree) of the corresponding integral Alexander modules, as well as the signed Euler characteristic property and generic vanishing for rank-one local systems on such subvarieties. Furthermore, we give a more conceptual (topological) interpretation of the signed Euler characteristic property in terms of vanishing of Novikov homology. As a byproduct, we prove a generic vanishing result for the $L^{2}$-Betti numbers of very affine manifolds. Our methods also recast June Huh's extension of Varchenko's conjecture to very affine manifolds, and provide a generalization of this result in the context of smooth closed sub-varieties of semi-abelian varieties.
\end{abstract}

\section{Contents}

1. Introduction

2. Topological preliminaries

2.1. Finite homotopy/homological type

2.2. Integral Alexander modules

2.3. Signed Euler characteristic property

2.4. Generic vanishing

2.5. Novikov-Betti and torsion numbers

2.6. $L^{2}$-Betti numbers

3. Non-proper Morse theory

3.1. Non-proper Morse theory after Palais-Smale

S

3.2. Circle-valued non-proper Morse theory

4. Topology of semi-abelian varieties. Statements of results

4.1. Subvarieties of semi-abelian varieties

4.2. Subvarieties of affine torus

4.3. Proof of Theorem 1.11

5. Semi-abelian varieites. Proof of structure results 19

5.1. Proof of Theorem 4.5 21

5.2. Proof of Proposition 4.6 23

6. Palais-Smale conditions $\quad 23$

References

Date: June 12, 2018.

2000 Mathematics Subject Classification. Primary 14K12, 20G20; Secondary 57D70.

Key words and phrases. semi-abelian variety, very affine manifold, non-proper Morse theory, generic vanishing, Alexander module, Novikov homology, $L^{2}$-Betti number. 


\section{INTRODUCTION}

A complex semi-abelian variety is a complex algebraic group $G$ which is an extension

$$
1 \rightarrow T \rightarrow G \rightarrow A \rightarrow 1
$$

where $A$ is an abelian variety and $T \cong\left(\mathbb{C}^{*}\right)^{m}$ is an algebraic torus. In this paper we study the topology of smooth closed subvarieties of semi-abelian varieties.

It was shown in [13] that for a smooth closed $n$-dimensional subvariety $X$ of a semi-abelian variety, the topological Euler characteristic of $X$ is signed, i.e.,

$$
(-1)^{n} \cdot \chi(X) \geq 0
$$

On the other hand, (11) fails to be true if $X$ is singular, e.g., see [2]. The proof of (11) in loc.cit. makes use of characteristic cycles and the language of perverse sheaves. In fact, (11) is a direct consequence of a more general fact that the Euler characteristic $\chi(G ; \mathcal{P})$ of a perverse sheaf on a semi-abelian variety is non-negative. In the case of abelian varieties, the latter also follows from the generic vanishing theorems for perverse sheaves, see e.g., [1, 27], while the corresponding statement for the Euler characteristic of perverse sheaves on a complex algebraic torus was proved in [20] (see also [15]).

One of the goals of this paper is to give a completely topological proof and interpretation of the signed Euler characteristic property (11). Our arguments are based on the non-proper Morse theory developed by Palais-Smale in [26], and should serve as an access point for geometers and topologists who are not necessarily accustomed with the language of perverse sheaves.

One of the main results of this paper is the following (see Corollary 4.7):

Theorem 1.1. Let $X$ be an $n$-dimensional closed connected smooth subvariety of a complex semiabelian variety. For a generic group homomorphism $\xi: \pi_{1}(X) \rightarrow \mathbb{Z}$, the corresponding infinite cyclic cover $X^{\xi}$ is homotopy equivalent to a finite $C W$-complex with possibly infinitely many $n$-cells attached. Moreover, if $\xi$ is surjective, then $X^{\xi}$ is connected.

An irreducible closed subvariety of a complex algebraic torus is called a very affine variety. These are heavily studied in tropical geometry and algebraic statistics, e.g., see [18]. Examples of very affine varieties include complements in the complex affine space of essential hyperplane arrangements, as well as complements of complex toric hyperplane arrangements. The signed Euler characteristic property for smooth very affine varieties was proved in [17] as a consequence of the generalized Varchenko conjecture; see also [2] for a different approach.

In the case of smooth very affine varieties, Theorem 1.1 can be refined as follows (see Corollary 4.14 and Corollary 4.17):

Theorem 1.2. Let $X$ be an $n$-dimensional smooth connected very affine variety in the complex affine torus $G$. Then for a generic homomorphism $\xi: \pi_{1}(X) \rightarrow \mathbb{Z}$, which factors through $\pi_{1}(X) \rightarrow$ $\pi_{1}(G)$, there exists a continuous map $F_{\xi}: X_{0} \rightarrow S^{1}$ defined on a subset $X_{0}$ of $X$, such that the following properties hold:

(1) $F_{\xi}$ is a fibration whose fiber is of finite homotopy type,

(2) $X$ is homotopy equivalent to $X_{0}$ with $(-1)^{n} \chi(X) n$-cells attached.

Moreover, if the mixed Hodge structure of $H^{1}(X ; \mathbb{Z})$ is pure of type $(1,1)$, or equivalently, there exists a smooth compactification $\bar{X}$ of $X$ such that $b_{1}(\bar{X})=0$, the above statement holds for any generic homomorphism $\xi: \pi_{1}(X) \rightarrow \mathbb{Z}$. 
The purity assumption from the statement of Theorem 1.2 is satisfied for the main examples of very affine varieties mentioned above: complements of essential hyperplane arrangements and resp. complements of toric hyperplane arrangements.

As one of the main topological applications of Theorem [1.1 we mention the following:

Theorem 1.3. (finite generation of integral Alexander modules)

Let $X$ be an $n$-dimensional closed connected smooth subvariety of a complex semi-abelian variety. For a generic group homomorphism $\xi: \pi_{1}(X) \rightarrow \mathbb{Z}$, the corresponding integral Alexander modules $H_{i}\left(X^{\xi} ; \mathbb{Z}\right)$ are finitely generated abelian groups for any $i \neq n$.

Theorem 1.3 implies immediately the following:

Corollary 1.4. (torsion property)

Let $X$ be an $n$-dimensional closed connected smooth subvariety of a complex semi-abelian variety. For a generic group homomorphism $\xi: \pi_{1}(X) \rightarrow \mathbb{Z}$, the corresponding complex Alexander modules $H_{i}\left(X^{\xi} ; \mathbb{C}\right)$ are torsion $\mathbb{C}\left[t, t^{-1}\right]$-modules, for all $i \neq n$.

Under the assumptions of the previous corollary, let $\Delta_{i}^{\xi}(t)$ denote the Alexander polynomial associated to $H_{i}\left(X^{\xi} ; \mathbb{C}\right)$. We also let $\Delta_{n}^{\xi}(t)$ be the Alexander polynomial associated to the torsion part of the finitely generated $\mathbb{C}\left[t, t^{-1}\right]$-module $H_{n}\left(X^{\xi} ; \mathbb{C}\right)$. Then by using [3, Proposition 1.4] we have the following:

Corollary 1.5. (roots of Alexander polynomials)

For any $i \geq 0$, the zeros of the Alexander polynomials $\Delta_{i}^{\xi}(t)$ are roots of unity.

Furthermore, Theorem 1.3 also has the following important consequences:

Corollary 1.6. (signed Euler characteristic)

If $X$ is an $n$-dimensional closed connected smooth subvariety of a complex semi-abelian variety, then $(-1)^{n} \cdot \chi(X) \geq 0$.

Corollary 1.7. (generic vanishing)

Let $X$ be an $n$-dimensional closed connected smooth subvariety of a complex semi-abelian variety, and fix a generic epimorphism $\xi: \pi_{1}(X) \rightarrow \mathbb{Z}$. Given any $s \in \mathbb{C}^{*}$, denote by $L_{s}^{\xi}$ the rank-one $\mathbb{C}$-local system on $X$ whose monodromy representation is given by

$$
\pi_{1}(X) \stackrel{\xi}{\rightarrow} \mathbb{Z} \rightarrow \mathbb{C}^{*}
$$

where the homomorphism $\mathbb{Z} \rightarrow \mathbb{C}^{*}$ is defined by $1 \mapsto s$. Then for all but finitely many $s \in \mathbb{C}^{*}$, we have:

$$
H_{i}\left(X, L_{s}^{\xi}\right)=0, \text { for all } i \neq n .
$$

A more conceptual (and purely topological) interpretation of the signed Euler characteristic property (1) can be given in terms of Novikov homology. More precisely, to any pair $(X, \xi)$ as above, one can associate Novikov-Betti numbers $b_{i}(X, \xi)$ and resp. Novikov-torsion numbers $q_{i}(X, \xi)$. Then the following result holds:

Corollary 1.8. (vanishing of Novikov homology)

Let $X$ be an $n$-dimensional closed connected smooth subvariety of a complex semi-abelian variety, and fix a generic epimorphism $\xi: \pi_{1}(X) \rightarrow \mathbb{Z}$ as above. Then $b_{i}(X, \xi)=q_{i}(X, \xi)=0$ for any $i \neq n$, and $b_{n}(X, \xi)=(-1)^{n} \chi(X)$. 
As an application of Theorem 1.2, we also obtain the following generic vanishing for the $L^{2}$ Betti numbers of very affine manifolds. To the authors' knowledge, this result seems to be new, and it provides a more unifying treatment of the corresponding assertions for toric arrangement complements, see [8] (which seems to contain a gap in the proof, cf. [6]) and, respectively, essential hyperplane arrangement complements, see [7].

If $\Gamma$ is a countable group, and the pair $(X, \xi)$ is as above, an epimorphism $\alpha: \pi_{1}(X) \rightarrow \Gamma$ is called $\xi$-admissible if $\xi$ factors through $\alpha$. Then we have:

Corollary 1.9. (Vanishing of $L^{2}$-Betti numbers of very affine manifolds)

Let $X$ be an $n$-dimensional smooth connected very affine variety in the complex affine torus $G$. Fix a generic epimorphism $\xi: \pi_{1}(X) \rightarrow \mathbb{Z}$, which factors through $\pi_{1}(X) \rightarrow \pi_{1}(G)$. Then, for any $\xi$-admissible epimorphism $\alpha: \pi_{1}(X) \rightarrow \Gamma$, we have that $b_{i}^{(2)}(X, \alpha)=0$ for all $i \neq n$, and $b_{n}^{(2)}(X, \alpha)=(-1)^{n} \chi(X)$.

Our methods also recast June Huh's extension of Varchenko's conjecture to very affine manifolds, see [17. Theorem 1.1], and provide a generalization of this result in the context of smooth closed sub-varieties of semi-abelian varieties.

Theorem 1.10. [17] Let $X$ be an n-dimensional closed connected smooth subvariety of $\left(\mathbb{C}^{*}\right)^{m}$, and let $\left(z_{1}, \ldots, z_{m}\right)$ be the coordinates of $\left(\mathbb{C}^{*}\right)^{m}$. Given $\mathbf{u}=\left(u_{1}, \ldots, u_{m}\right) \in \mathbb{Z}^{m}$, let $\psi_{\mathbf{u}}$ be the restriction of the master function $z_{1}^{u_{1}} \cdots z_{m}^{u_{m}}$ to $X$. If $\mathbf{u}$ is sufficiently general, then the following properties hold:

(1) The function $\psi_{\mathbf{u}}$ has only finitely many critical points on $X$.

(2) All critical points of $\psi_{\mathbf{u}}$ are regular.

(3) The number of critical points is equal to the signed Euler characteristic

$$
(-1)^{n} \chi(X) \text {. }
$$

Notice that the function $\psi_{\mathbf{u}}$ has a critical point at $x \in X$ if and only if the logarithmic 1-form

$$
\frac{d \psi_{\mathbf{u}}}{\psi_{\mathbf{u}}}=u_{1} \frac{d z_{1}}{z_{1}}+\cdots+u_{m} \frac{d z_{m}}{z_{m}}
$$

degenerates at $x$. Moreover, $\psi_{\mathbf{u}}$ has a regular critical point at $x$ if and only if $\frac{d \psi_{\mathbf{u}}}{\psi_{\mathbf{u}}}$ has a regular singularity at $x$.

The logarithmic forms $\frac{d \psi_{\mathbf{u}}}{\psi_{\mathbf{u}}}$ are well-defined for $\mathbf{u} \in \mathbb{C}^{m}$, and they are left invariant holomorphic 1 -forms on $\left(\mathbb{C}^{*}\right)^{m}$. Conversely, every left invariant holomorphic 1 -form on $\left(\mathbb{C}^{*}\right)^{m}$ is equal to $\frac{d \psi_{\mathbf{u}}}{\psi_{\mathbf{u}}}$ for some $\mathbf{u} \in \mathbb{C}^{m}$. Thus, Theorem 1.10 can be formulated by using left invariant holomorphic 1 -forms on the affine torus. In this setting, we obtain the following generalization of Huh's theorem to smooth subvarieties of semi-abelian varieties.

Theorem 1.11. Let $G$ be a complex semi-abelian variety, and let $X$ be an $n$-dimensional closed connected smooth subvariety of $G$. Let $\Gamma$ be the space of left invariant holomorphic 1-forms on $G$. Given $\eta \in \Gamma$, we denote the restriction of $\eta$ to $X$ by $\eta_{X}$. If $\eta \in \Gamma$ is general, then the following statements hold:

(1) The holomorphic 1-form $\eta_{X}$ degenerates at finitely many points in $X$.

(2) At all degeneration points, $\eta_{X}$ has regular singularity.

(3) The number of degeneration points of $\eta_{X}$ is equal to the signed Euler characteristic

$$
(-1)^{n} \chi(X) \text {. }
$$


Note that the signed Euler characteristic $(-1)^{n} \chi(X)$ of $X$ as in the above theorem can also be identified with the Gaussian degree of $X$, see [13][Corollary 1.5].

Let us briefly describe the structure of the paper. In Section 2 we develop the topological tools needed for proving Theorem 1.3 and its consequences (Corollaries 1.6] 1.9). In Section 3 we recall the main aspects of the non-proper Morse theory of Palais-Smale, and provide a circle-valued version of this theory. In Section 4 we introduce the main constructions of the paper, and prove Theorem 1.1 (see Corollary 4.7), Theorem 1.2 (see Corollary 4.14 and Corollary 4.17) and Theorem 1.11. Sections 5 and 6 are of technical nature, and supply the proofs of the Morse-theoretic statements behind Theorems [1.1, 1.2 and 1.11.

\section{TOPOLOGICAL PRELIMINARIES}

In this section we collect the topological facts needed to prove Theorem 1.3 and its consequences. The results presented here are often formulated in greater generality than needed in the subsequent sections, as they may be of independent interest.

Throughout this paper, all topological spaces under consideration are homotopy equivalent to CW complexes.

\subsection{Finite homotopy/homological type.}

Definition 2.1. Let $X$ be a connected topological space. We say that:

(1) $X$ has finite $k$-homotopy type if $X$ is homotopy equivalent to a CW complex with finite $k$-skeleton.

(2) $X$ has finite homotopy type if $X$ is homotopy equivalent to a finite CW complex.

(3) $X$ has finite $k$-homological type if $H_{i}(X ; \mathbb{Z})$ is a finitely generated abelian group for any $i \leq k$.

(4) $X$ has finite homological type if $H_{i}(X ; \mathbb{Z})$ is a finitely generated abelian group for any $i \geq 0$.

Remark 2.2. Note that if $X$ has finite $k$-homotopy type, then $X$ has finite $k$-homological type. However, the converse fails to be true.

2.2. Integral Alexander modules. Let $X$ be a connected topological space of finite homotopy type, and $\xi: \pi_{1}(X) \rightarrow \mathbb{Z}$ a non-zero homomorphism. Denote by $X^{\xi}$ the infinite cyclic cover of $X$ defined by $\operatorname{Ker}(\xi)$. Then $X^{\xi}$ is the disjoint union of $[\mathbb{Z}:$ Image $(\xi)]$ homeomorphic connected components. The group of covering transformations of the cover $X^{\xi}$ is isomorphic to $\mathbb{Z}$, so the singular chain (or cellular) groups $C_{i}\left(X^{\xi} ; \mathbb{Z}\right)$ and homology groups $H_{i}\left(X^{\xi} ; \mathbb{Z}\right)$ become modules over the group ring $\mathbb{Z}[\mathbb{Z}] \cong \mathbb{Z}\left[t^{ \pm 1}\right]$.

Definition 2.3. The $\mathbb{Z}\left[t^{ \pm 1}\right]$-module $H_{i}\left(X^{\xi} ; \mathbb{Z}\right)$ is called the $i$-th integral Alexander module of the pair $(X, \xi)$.

For future reference, we note that $\xi$ can be regarded as an element in $H^{1}(X ; \mathbb{Z})$ via the canonical identification:

$$
\operatorname{Hom}\left(\pi_{1}(X), \mathbb{Z}\right) \cong \operatorname{Hom}\left(H_{1}(X), \mathbb{Z}\right) \cong H^{1}(X ; \mathbb{Z}) .
$$

Moreover, by the representability theorem for cohomology, any such class $\xi \in H^{1}(X ; \mathbb{Z})$ is represented by a homotopy class of maps $X \rightarrow S^{1}$. Let $f_{\xi}: X \rightarrow S^{1}$ be a fixed homotopy representative for $\xi$. 
For any subspace $Z$ of $X$ with inclusion map $j: Z \hookrightarrow X$, we denote by $Z^{\xi}$ the infinite cyclic cover of $Z$ defined by the composite homomorphism

$$
\xi \circ j_{*}: \pi_{1}(Z) \stackrel{j_{*}}{\rightarrow} \pi_{1}(X) \stackrel{\xi}{\rightarrow} \mathbb{Z} .
$$

Note that this homomorphism is induced by $\left.f_{\xi}\right|_{Z}: Z \rightarrow S^{1}$.

With the above notations, we have the following preliminary result:

Proposition 2.4. Let $X$ be a connected topological space of finite homotopy type, and fix a non-zero homomorphism $\xi: \pi_{1}(X) \rightarrow \mathbb{Z}$.

(a) Assume that $X$ is obtained up to homotopy from a subspace $Z$, by attaching cells of dimension $\geq n$ (with $n \geq 2$ ). Moreover, assume that the infinite cyclic cover $Z^{\xi}$ has finite $(n-1)$-homological type. Then the infinite cyclic cover $X^{\xi}$ of $X$ has finite $(n-1)$ homological type, i.e., the integral Alexander modules $H_{i}\left(X^{\xi} ; \mathbb{Z}\right)$ are finitely generated abelian groups for any $i<n$.

(b) If $X$ is obtained from $Z$ by attaching cells of dimension exactly $n$ and $Z^{\xi}$ has finite homological type, then $H_{i}\left(X^{\xi} ; \mathbb{Z}\right)$ is a finitely generated abelian group for any $i \neq n$.

Proof. Without loss of generality, we may assume that $X$ and $Z$ are CW complexes. Let $j: Z \hookrightarrow X$ denote as before the inclusion map. Since $X \backslash Z$ contains only cells of dimension $\geq n$, the pair $(X, Z)$ is $(n-1)$-connected. So the homomorphism $j_{*}: \pi_{1}(Z) \rightarrow \pi_{1}(X)$ induced by inclusion is onto if $n=2$ and it is an isomorphism if $n>2$.

(a) If $X$ is obtained from $Z$ by adding cells of dimension $\geq n$, the same is true for the infinite cyclic covers $X^{\xi}$ and $Z^{\xi}$ defined by $\xi$ and $\xi \circ j_{*}$, respectively. It follows that the induced group homomorphism

$$
H_{i}\left(Z^{\xi} ; \mathbb{Z}\right) \rightarrow H_{i}\left(X^{\xi} ; \mathbb{Z}\right)
$$

is an isomorphism for $i<n-1$ and it is onto for $i=n-1$. The result follows now from the fact that $Z^{\xi}$ has finite $(n-1)$-homological type.

(b) If $X$ is obtained from $Z$ by attaching cells of dimension exactly $n$, the same is true for the infinite cyclic covers $X^{\xi}$ and $Z^{\xi}$. From the homology long exact sequence of the pair $\left(X^{\xi}, Z^{\xi}\right)$, it follows that the induced group homomorphism

$$
H_{i}\left(Z^{\xi} ; \mathbb{Z}\right) \rightarrow H_{i}\left(X^{\xi} ; \mathbb{Z}\right)
$$

is an isomorphism for $i<n-1$ and $i>n$, and it is onto for $i=n-1$. The claim follows since $Z^{\xi}$ has finite homological type.

Remark 2.5. As it can be easily seen from the above proof, the conclusions of Proposition 2.4 still hold if we only assume that the infinite cyclic cover $X^{\xi}$ is built (up to homotopy) from a subspace of finite homological type by attaching (possibly infinitely many) cells of dimension $\geq n$, and resp. $n$. This is in fact the case for smooth closed subvarieties of complex semiabelian varieties (see Theorem 1.1). In particular, this observation yields a proof of Theorem 1.3 from the Introduction.

Remark 2.6. When the integral Alexander module $H_{i}\left(X^{\xi} ; \mathbb{Z}\right)$ is a torsion $\mathbb{Z}\left[t, t^{-1}\right]$-module, it is not necessarily finitely generated as a $\mathbb{Z}$-module. For example, as noted in [24, section 2], the integral Alexander module of the complement $X$ in $S^{3}$ of the knot $5_{2}$ is $\mathbb{Z}\left[t, t^{-1}\right] /\left(2 t^{2}-3 t+2\right)$, which is torsion over $\mathbb{Z}\left[t, t^{-1}\right]$, but not finitely generated over $\mathbb{Z}$. 
We next give a concrete geometric situation when the assumptions of Proposition2.4 are fullfilled. We begin with the following simple observation.

Lemma 2.7. Let $f: X \rightarrow S^{1}$ be a locally trivial topological fibration with fiber $F$ of finite $k$ homological type, and let $\xi:=f_{*}: \pi_{1}(X) \rightarrow \pi_{1}\left(S^{1}\right) \cong \mathbb{Z}$. Then $X^{\xi}$ has finite $k$-homological type, i.e., $H_{i}\left(X^{\xi} ; \mathbb{Z}\right)$ is a finitely generated abelian group for any $i \leq k$. If, moreover, the fiber $F$ has finite homological type, then so does $X^{\xi}$.

Proof. The infinite cyclic cover $X^{\xi}$ is homeomorphic to $F \times \mathbb{R}$, hence homotopy equivalent to $F$, with compatible $\mathbb{Z}$-actions. It follows that for any $i \geq 0$, we have an isomorphism of abelian groups (and $\mathbb{Z}\left[t^{ \pm 1}\right]$-modules):

$$
H_{i}\left(X^{\xi} ; \mathbb{Z}\right) \cong H_{i}(F ; \mathbb{Z})
$$

which yields the claim.

As an immediate consequence of Lemma 2.7 and Proposition 2.4, we have the following:

Proposition 2.8. Let $X$ be a connected topological space of finite homotopy type, and fix a homomorphism $\xi: \pi_{1}(X) \rightarrow \mathbb{Z}$ with a homotopy representative $f_{\xi}: X \rightarrow S^{1}$.

(a) Assume that $X$ is obtained from a subspace $Z$, by attaching cells of dimension $\geq n$ (with $n \geq 2$ ). Moreover, assume that $\left.f_{\xi}\right|_{Z}: Z \rightarrow S^{1}$ is a locally trivial topological fibration whose fiber $F$ has finite $(n-1)$-homological type. Then the infinite cyclic cover $X^{\xi}$ of $X$ has finite $(n-1)$-homological type.

(b) If $X$ is obtained from $Z$ by attaching cells of dimension exactly $n$, and the fiber of the fibration $\left.f_{\xi}\right|_{Z}: Z \rightarrow S^{1}$ has finite homological type, then $H_{i}\left(X^{\xi} ; \mathbb{Z}\right)$ is a finitely generated abelian group for any $i \neq n$.

Example 2.9. As main examples of topological spaces satisfying the assumptions of Proposition 2.8 , we mention the following:

(1) complements in $\mathbb{C}^{n}$ of hypersurfaces in general position (or regular) at infinity, e.g., see [22, 10]; here, if $X=\mathbb{C}^{n} \backslash\{f=0\}$, we can take $\xi=f_{*}$, or any other positive homomorphism; the fibration structure corresponds in this case to the complement of the link at infinity.

(2) complements of essential hyperplane arrangements in $\mathbb{C}^{n}$, with $\xi$ a positive (rank 1 ) homomorphism, e.g., see [9, 19]; the fibration structure is given in this case by the boundary manifold of the arrangement.

(3) smooth connected very affine varieties, see Theorem 1.2 note that essential hyperplane arrangement complements in $\mathbb{C}^{n}$, as well as complements of toric hyperplane arrangements (e.g., studied in [4, 5]) are examples of smooth very affine varieties.

Moreover, in all these cases, the space $X$ under cosideration is built from a fibration over the circle $S^{1}$ by adding $|\chi(X)|$ cells of middle (real) dimension $n$.

\subsection{Signed Euler characteristic property.}

Proposition 2.10. Let $X$ be a connected topological space of finite homotopy type, and fix a homomorphism $\xi: \pi_{1}(X) \rightarrow \mathbb{Z}$. Assume that $X$ is obtained up to homotopy from a subspace $Z$, by attaching $\ell$ cells of dimension exactly $n$. Moreover, assume that the infinite cyclic cover $Z^{\xi}$ has finite homological type. Then

$$
(-1)^{n} \chi(X)=\ell \geq 0
$$


Proof. Let $H_{i}\left(X^{\xi} ; \mathbb{C}\right)$ denote the $i$-th complex Alexander module of the pair $(X, \xi)$. It follows from Proposition 2.4 (b) that $H_{i}\left(X^{\xi} ; \mathbb{C}\right)$ is a torsion $\mathbb{C}\left[t, t^{-1}\right]$-module, for all $i \neq n$. Since the $\mathbb{C}\left[t, t^{-1}\right]$-ranks of the complex Alexander modules can be used to compute the Euler characteristic of $X$, we then have that:

$$
\chi(X)=(-1)^{n} \operatorname{rank}_{\mathbb{C}\left[t, t^{-1}\right]} H_{n}\left(X^{\xi} ; \mathbb{C}\right),
$$

which yields the signed Euler characteristic property. Similarly, it follows by our assumption that the complex Alexander modules $H_{i}\left(Z^{\xi} ; \mathbb{C}\right)$ are torsion over $\mathbb{C}\left[t, t^{-1}\right]$, for all $i \geq 0$. The fact that rank $\mathbb{C}_{\left.\mathbb{C} t, t^{-1}\right]} H_{n}\left(X^{\xi} ; \mathbb{C}\right)=\ell$ follows now from the homology long exact sequence of the pair $\left(X^{\xi}, Z^{\xi}\right)$, by noting that $H_{n}\left(X^{\xi}, Z^{\xi} ; \mathbb{C}\right) \cong \mathbb{C}\left[t, t^{-1}\right]^{\oplus \ell}$, and $H_{i}\left(X^{\xi}, Z^{\xi} ; \mathbb{C}\right)=0$ for all $i \neq n$.

In view of Remark 2.5 and Theorem 1.1. Proposition 2.10 yields the signed Euler characteristic property (1) for smooth closed subvarieties of semi-abelian varieties, i.e., a proof of Corollary 1.6.

\subsection{Generic vanishing. The following is an application of Propositions 2.4 and 2.8 .}

Proposition 2.11. Let $X$ be a connected topological space of finite homotopy type, with an epimorphism $\xi: \pi_{1}(X) \rightarrow \mathbb{Z}$. Assume that the associated infinite cyclic cover $X^{\xi}$ of $X$ has finite $k$-homological type. Given any $s \in \mathbb{C}^{*}$, denote by $L_{s}^{\xi}$ the rank-one $\mathbb{C}$-local system on $X$ whose monodromy representation is given by

$$
\pi_{1}(X) \stackrel{\xi}{\rightarrow} \mathbb{Z} \rightarrow \mathbb{C}^{*}
$$

where the homomorphism $\mathbb{Z} \rightarrow \mathbb{C}^{*}$ is defined by $1 \mapsto s$. Then for all but finitely many $s \in \mathbb{C}^{*}$, we have:

$$
H_{i}\left(X, L_{s}^{\xi}\right)=0, \text { for all } i \leq k .
$$

Proof. Consider the Milnor long exact sequence (e.g., see [11, Theorem 4.2]):

$$
\cdots \longrightarrow H_{i}\left(X^{\xi} ; \mathbb{C}\right) \stackrel{t-s}{\longrightarrow} H_{i}\left(X^{\xi} ; \mathbb{C}\right) \longrightarrow H_{i}\left(X, L_{s}^{\xi}\right) \longrightarrow H_{i-1}\left(X^{\xi} ; \mathbb{C}\right) \longrightarrow \cdots
$$

Under our assumptions, $H_{i}\left(X^{\xi} ; \mathbb{C}\right)$ is a finitely generated torsion $\mathbb{C}\left[t, t^{-1}\right]$-module for any $i \leq k$. Hence multiplication by $t-s$ on $H_{i}\left(X^{\xi} ; \mathbb{C}\right.$ ) is an isomorphism (for $i \leq k$ ) provided that $s$ is not a root of the Alexander polynomial associated to $H_{i}\left(X^{\xi} ; \mathbb{C}\right)$. The claim follows.

In the context of Proposition 2.4 (b) and in the notations of the previous Proposition, we also have the following:

Proposition 2.12. Let $X$ be a connected topological space of finite homotopy type, with an epimorphism $\xi: \pi_{1}(X) \rightarrow \mathbb{Z}$. Assume that $X$ is obtained up to homotopy from a subspace $Z$, by attaching cells of dimension exactly $n$. Moreover, assume that the infinite cyclic cover $Z^{\xi}$ has finite homological type. Then for all but finitely many $s \in \mathbb{C}^{*}$, we have:

$$
H_{i}\left(X, L_{s}^{\xi}\right)=0, \text { for all } i \neq n \text {. }
$$

Proof. Proposition 2.4(b) yields that the complex Alexander modules $H_{i}\left(X^{\xi} ; \mathbb{C}\right)$ are torsion $\mathbb{C}\left[t, t^{-1}\right]$ modules, for all $i \neq n$. Let $\Delta_{i}^{\xi}(t)$ denote the associated Alexander polynomial. We also let $\Delta_{n}^{\xi}(t)$ be the Alexander polynomial associated to the torsion part of the finitely generated $\mathbb{C}\left[t, t^{-1}\right]$-module $H_{n}\left(X^{\xi} ; \mathbb{C}\right)$.

By using the above Milnor long exact sequence, it follows as in [11, Theorem 4.2], that for any $i \geq 0$, we have:

$$
\operatorname{dim} H_{i}\left(X, L_{s}^{\xi}\right)=\operatorname{rank}_{\mathbb{C}\left[t, t^{-1}\right]} H_{i}\left(X^{\xi} ; \mathbb{C}\right)+N(s, i)+N(s, i-1)
$$


where $N(s, i)$ is the number of direct summands in the $(t-s)$-torsion of the $\mathbb{C}\left[t, t^{-1}\right]$-module $H_{i}\left(X^{\xi} ; \mathbb{C}\right)$. So, as long as $s \in \mathbb{C}^{*}$ is chosen such that $H_{i}\left(X^{\xi} ; \mathbb{C}\right)$ has no $(t-s)$-torsion for any $i \geq 0$ (or, equivalently, $s$ is not a root of any of the Alexander polynomials $\Delta_{i}^{\xi}(t)$, for all $i \geq 0)$, we have that $H_{i}\left(X, L_{s}^{\xi}\right)=0$, for all $i \neq n$ and $\operatorname{dim} H_{n}\left(X, L_{s}^{\xi}\right)=\operatorname{rank}_{\mathbb{C}\left[t, t^{-1}\right]} H_{n}\left(X^{\xi} ; \mathbb{C}\right)=$ $(-1)^{n} \chi(X)$.

Remark 2.13. As in Remark 2.5, the generic vanishing of Proposition 2.12 still holds if we only assume that $X^{\xi}$ is built (up to homotopy) from a subspace of finite homological type by adding (possibly infinitely many) cells of dimension $n$. In particular, in view of Theorem 1.1, this yields a proof of Corollary 1.7 from the Introduction.

2.5. Novikov-Betti and torsion numbers. Finiteness properties of infinite cyclic covers translate into vanishing of the corresponding Novikov-Betti and resp. Novikov-torsion numbers. For the convenience of the reader, we include here the relevant definitions.

Let $X$ be a connected topological space of finite homotopy type, and fix $\xi \in H^{1}(X ; \mathbb{R})$. Via the canonical identification

$$
H^{1}(X ; \mathbb{R}) \cong \operatorname{Hom}\left(H_{1}(X), \mathbb{R}\right) \cong \operatorname{Hom}\left(\pi_{1}(X), \mathbb{R}\right)
$$

any real cohomology class $\xi \in H^{1}(X ; \mathbb{R})$ determines a homomorphism $\xi: \pi_{1}(X) \rightarrow \mathbb{R}$, where $\mathbb{R}$ is considered as a group with the usual addition. (We use here the same notation for the cohomology class and the corresponding homomorphism on the fundamental group; it should be clear from the context which one is referred to.) Let $\Gamma_{\xi}$ denote the image of the homomorphism $\xi$. Then $\xi$ factors as $\xi=i_{\xi} \circ \eta_{\xi}$, with $\eta_{\xi}: \pi_{1}(X) \rightarrow \Gamma_{\xi}$ an epimorphism and $i_{\xi}: \Gamma_{\xi} \hookrightarrow \mathbb{R}$ a monomorphism. The group $\Gamma_{\xi}$ is a finitely generated free abelian group, say $\Gamma_{\xi} \cong \mathbb{Z}^{r}, r \geq 0$. The integer $r$ is called the rank of $\xi$. Note that if $\xi \in H^{1}(X ; \mathbb{Z})$ is an integral cohomology class, then it has rank 1 . The set of rank 1 classes is dense in $H^{1}(X ; \mathbb{R})$.

Let

$$
X^{\xi} \longrightarrow X
$$

be the covering of $X$ defined by $\operatorname{Ker}(\xi)$. The group of covering transformations of $X^{\xi}$ is isomorphic to $\Gamma_{\xi}$, so the cellular groups $C_{i}\left(X^{\xi} ; \mathbb{Z}\right)$, as well as the homology groups $H_{i}\left(X^{\xi} ; \mathbb{Z}\right.$ ), become (finitely generated) left modules over the group ring $\mathbb{Z} \Gamma_{\xi} \cong \mathbb{Z}\left[t_{1}^{ \pm 1}, \ldots, t_{r}^{ \pm 1}\right]$. Let $Q_{\xi}$ denote the field of fractions of $\mathbb{Z} \Gamma_{\xi}$.

Definition 2.14. The $i$-th Novikov-Betti number $b_{i}(X, \xi)$ of the pair $(X, \xi)$ is the $\mathbb{Z} \Gamma_{\xi}$-rank of the module $H_{i}\left(X^{\xi} ; \mathbb{Z}\right)$, i.e., the dimension of the $Q_{\xi}$-vector space $Q_{\xi} \otimes_{\mathbb{Z} \Gamma_{\xi}} H_{i}\left(X^{\xi} ; \mathbb{Z}\right)$.

Remark 2.15. Note that if $\xi \in H^{1}(X ; \mathbb{Z})$ is an integral cohomology class corresponding to an epimorphism $\xi: \pi_{1}(X) \rightarrow \mathbb{Z}$, then the $i$-th Novikov-Betti number $b_{i}(X, \xi)$ is just the $\mathbb{Z}\left[t^{ \pm 1}\right]$-rank of the integral Alexander module $H_{i}\left(X^{\xi} ; \mathbb{Z}\right)$.

Alternatively, following [12], one can define the Novikov-Betti numbers of $(X, \xi)$ as ranks over the rational Novikov ring of $\Gamma_{\xi}$, which is a certain localization of $\mathbb{Z} \Gamma_{\xi}$. In more detail, a Laurent polynomial $p=\sum_{\gamma} n_{\gamma} \gamma \in \mathbb{Z} \Gamma_{\xi}$ is called monic if $n_{e}=1$ and if for all $\gamma \neq e$ with $n_{\gamma} \neq 0$ we have $i_{\xi}(\gamma)<0$. Here $e$ is the identity element in the group $\Gamma_{\xi}$, hence also the multiplicative identity element in the ring $\mathbb{Z} \Gamma_{\xi}$. Denote by $S_{\xi}$ the set of all monic polynomials in $\mathbb{Z} \Gamma_{\xi}$.

Definition 2.16. The rational Novikov ring $\mathcal{R} \Gamma_{\xi}$ of $\Gamma_{\xi}$ is defined as the localization of $\mathbb{Z} \Gamma_{\xi}$ at the multiplicative set $S_{\xi}$, that is,

$$
\mathcal{R} \Gamma_{\xi}:=S_{\xi}^{-1} \mathbb{Z} \Gamma_{\xi}
$$


The advantage of working over the rational Novikov ring is that, as shown in [12, Lemma 1.15], the ring $\mathcal{R} \Gamma_{\xi}$ is a PID. Next note that

$$
S_{\xi}^{-1} C_{*}\left(X^{\xi}\right):=S_{\xi}^{-1} \mathbb{Z} \Gamma_{\xi} \otimes_{\mathbb{Z} \Gamma_{\xi}} C_{*}\left(X^{\xi}\right) \cong \mathcal{R} \Gamma_{\xi} \otimes_{\mathbb{Z} \pi_{1}(X)} C_{*}(\tilde{X}),
$$

where $\tilde{X}$ denotes the universal cover of $X$. Hence, by the exactness of the localization functor, one has an isomorphism of $\mathcal{R} \Gamma_{\xi}$-modules:

$$
H_{*}\left(X ; \mathcal{R} \Gamma_{\xi}\right) \cong S_{\xi}^{-1} H_{*}\left(X^{\xi} ; \mathbb{Z}\right) .
$$

It follows that the $i$-th Novikov-Betti number of $(X, \xi)$ equals

$$
b_{i}(X, \xi)=\operatorname{rank}_{\mathcal{R} \Gamma_{\xi}} H_{i}\left(X ; \mathcal{R} \Gamma_{\xi}\right) .
$$

Definition 2.17. The $i$-th Novikov torsion number $q_{i}(X, \xi)$ is defined as the minimal number of generators of the torsion submodule of the $\mathcal{R} \Gamma_{\xi}$-module $H_{i}\left(X ; \mathcal{R} \Gamma_{\xi}\right)$.

The following result is well-known, e.g., see [12, Chapter 1]:

Proposition 2.18. For any $\xi \in H^{1}(X ; \mathbb{R})$, the Euler-Poincaré characteristic of $X$ is computed as:

$$
\chi(X)=\sum_{i \geq 0}(-1)^{i} \cdot b_{i}(X, \xi) .
$$

We also recall here the following simple but useful fact, see [12, Proposition 1.35]:

Proposition 2.19. Suppose that $\xi \in H^{1}(X ; \mathbb{R}), \xi \neq 0$, and the $i$-th homology $H_{i}\left(X^{\xi} ; \mathbb{Z}\right)$ is finitely generated as an abelian group. Then $b_{i}(X, \xi)=q_{i}(X, \xi)=0$.

In particular, this result applies in the context of Propositions 2.4 and 2.8. Namely, Proposition 2.4 yields the following result (compare also with [14, 19] for special cases):

Corollary 2.20. Let $X$ be a connected topological space of finite homotopy type, and fix a (nonzero) epimorphism $\xi: \pi_{1}(X) \rightarrow \mathbb{Z}$.

(a) Assume that $X$ is obtained up to homotopy from a subspace $Z$, by attaching cells of dimension $\geq n$ (with $n \geq 2$ ). Moreover, assume that the infinite cyclic cover $Z^{\xi}$ defined by the kernel of the composition $\pi_{1}(Z) \rightarrow \pi_{1}(X) \stackrel{\xi}{\rightarrow} \mathbb{Z}$ has finite $(n-1)$-homological type. Then, for any $i<n$, we have that $b_{i}(X, \xi)=0$ and $q_{i}(X, \xi)=0$.

(b) If $X$ is obtained from $Z$ by attaching cells of dimension exactly $n$ and $Z^{\xi}$ has finite homological type, then $b_{i}(X, \xi)=q_{i}(X, \xi)=0$ for any $i \neq n$, and $b_{n}(X, \xi)=(-1)^{n} \chi(X)$.

Note that Theorem 1.3 together with Propositions 2.18 and 2.19 yields now a proof of Corollary 1.8

2.6. $L^{2}$-Betti numbers. Novikov-Betti numbers can be regarded as special cases of $L^{2}$-Betti numbers. In fact, the following identification holds:

$$
b_{i}(X ; \xi)=b_{i}^{(2)}\left(X, \xi: \pi_{1}(X) \rightarrow \operatorname{Image}(\xi)\right)
$$

However, Novikov torsion numbers do not have such interpretation in terms of $L^{2}$-invariants. For completeness, we include here the definition of $L^{2}$-Betti numbers.

Let $\Gamma$ be a countable group, with

$$
l^{2}(\Gamma):=\left\{f:\left.\Gamma \rightarrow \mathbb{C}\left|\sum_{g \in \Gamma}\right| f(g)\right|^{2}<\infty\right\}
$$


the Hilbert space of square-summable functions on $\Gamma$. Then $\Gamma$ acts on $l^{2}(\Gamma)$ by right multiplication, so $\mathbb{C}[\Gamma]$ can be regarded as a subset of $\mathcal{B}\left(l^{2}(\Gamma)\right)$, the set of bounded operators on $l^{2}(\Gamma)$. The von Neumann algebra $\mathcal{N}(\Gamma)$ of $\Gamma$ is defined as the closure of $\mathbb{C}[\Gamma] \subset \mathcal{B}\left(l^{2}(\Gamma)\right)$ with respect to pointwise convergence in $\mathcal{B}\left(l^{2}(\Gamma)\right)$. Any $\mathcal{N}(\Gamma)$-module $\mathcal{M}$ has a dimension $\operatorname{dim}_{\mathcal{N}(\Gamma)}(\mathcal{M}) \in \mathbb{R}_{\geq 0} \cup\{\infty\}$. We refer to [21, Def.6.50] for details.

Definition 2.21. To any connected topological space $X$ of finite homotopy type and a group homomorphism $\alpha: \pi_{1}(X) \rightarrow \Gamma$, we associate $L^{2}$-Betti numbers

$$
b_{i}^{(2)}(X, \alpha):=\operatorname{dim}_{\mathcal{N}(\Gamma)} H_{i}(X ; \mathcal{N}(\Gamma)):=\operatorname{dim}_{\mathcal{N}(\Gamma)} H_{i}\left(C_{*}\left(X^{\Gamma}\right) \otimes_{\mathbb{Z} \Gamma} \mathcal{N}(\Gamma)\right) \in[0, \infty],
$$

where $X^{\Gamma}$ is the regular covering of $X$ defined by $\operatorname{Ker}(\alpha)$, and $C_{*}\left(X^{\Gamma}\right)$ is the singular chain complex of $X^{\Gamma}$ with (right) $\Gamma$-action given by covering translations. If $X$ is a $C W$ complex, the cellular chain complex of $X^{\Gamma}$ can be used in the above definition of $L^{2}$-Betti numbers.

Remark 2.22. Since

$$
b_{i}^{(2)}\left(X, \alpha: \pi_{1}(X) \rightarrow \Gamma\right)=b_{i}^{(2)}\left(X, \alpha: \pi_{1}(X) \rightarrow \operatorname{Image}(\alpha)\right),
$$

we can assume without any loss of generality that $\alpha$ is an epimorphism.

The following properties of $L^{2}$-Betti numbers are standard, we refer to [21] for details:

Lemma 2.23. For any connected topological space $X$ and group homomorphism $\alpha: \pi_{1}(X) \rightarrow \Gamma$,

(a) $b_{i}^{(2)}(X, \alpha)$ is a homotopy invariant of the pair $(X, \alpha)$.

(b) $b_{0}^{(2)}(X, \alpha)=0$ if $\Gamma$ is infinite and $b_{0}^{(2)}(X, \alpha)=\frac{1}{|\Gamma|}$ if $\Gamma$ is finite.

(c) if $X$ is of finite homotopy type, then

$$
\chi(X)=\sum_{i \geq 0}(-1)^{i} b_{i}^{(2)}(X, \alpha) .
$$

Remark 2.24. Since all spaces we work with are homotopy equivalent to CW complexes, we can assume, without loss of generality, that all spaces in this section are CW-complexes.

Let us now assume that $X$ is a finite CW complex obtained from a CW (sub)-complex $Z$ by attaching cells of dimension $\geq n$. Let $\alpha_{Z}$ be the composition

$$
\alpha_{Z}: \pi_{1}(Z) \stackrel{j_{*}}{\longrightarrow} \pi_{1}(X) \stackrel{\alpha}{\longrightarrow} \Gamma,
$$

and let $Z^{\Gamma}$ be the corresponding $\Gamma$-cover of $Z$. Let $b_{i}^{(2)}\left(Z, \alpha_{Z}\right)$ denote the $L^{2}$-Betti numbers of the pair $\left(Z, \alpha_{Z}\right)$. Then the following holds:

Proposition 2.25. Assume that $X$ is a finite CW complex obtained from a CW (sub)-complex $Z$ by attaching cells of dimension $\geq n$. Then for any homomorphism $\alpha: \pi_{1}(X) \rightarrow \Gamma$ we have:

$$
b_{i}^{(2)}(X, \alpha)=b_{i}^{(2)}\left(Z, \alpha_{Z}\right), \text { for } i<n-1,
$$

and

$$
b_{n-1}^{(2)}(X, \alpha) \leq b_{n-1}^{(2)}\left(Z, \alpha_{Z}\right) .
$$

If, moreover, $X$ is obtained from $Z$ by attaching cells of dimension exactly $n$, then we also have the equalities

$$
b_{i}^{(2)}(X, \alpha)=b_{i}^{(2)}\left(Z, \alpha_{Z}\right), \text { for } i>n \text {. }
$$


Proof. Since $X$ is obtained from $Z$ by adding cells of dimension $\geq n$, the same is true for their corresponding $\Gamma$-coverings $X^{\Gamma}$ and $Z^{\Gamma}$, respectively. In particular, $C_{i}\left(Z^{\Gamma}\right)=C_{i}\left(X^{\Gamma}\right)$ for $i<n$. It follows that $H_{i}(Z ; \mathcal{N}(\Gamma)) \cong H_{i}(X ; \mathcal{N}(\Gamma))$ for $i<n-1$, and the induced homomorphism

$$
H_{n-1}(Z ; \mathcal{N}(\Gamma)) \longrightarrow H_{n-1}(X ; \mathcal{N}(\Gamma))
$$

is surjective. The first claim follows now from [21, Thm.6.7].

If, moreover, $X$ is obtained from $Z$ by attaching cells of dimension exactly $n$, then $C_{i}(Z, \mathcal{N}(\Gamma))=$ $C_{i}(X, \mathcal{N}(\Gamma))$ for any $i \neq n$, and $C_{n}(X, \mathcal{N}(\Gamma))=C_{n}(Z, \mathcal{N}(\Gamma)) \oplus P$, where $P$ is the free $\mathcal{N}(\Gamma)$ module generated by the extra $n$-cells of $X$. Here, we use the notation $C_{i}(X, \mathcal{N}(\Gamma)):=C_{i}\left(X^{\Gamma}\right) \otimes_{\mathbb{Z} \Gamma}$ $\mathcal{N}(\Gamma)$. An easy diagram chase then yields the isomorphisms $H_{i}(Z ; \mathcal{N}(\Gamma)) \cong H_{i}(X ; \mathcal{N}(\Gamma))$ for all $i>n$. This completes the proof.

The $L^{2}$-Betti numbers provide obstructions for a space to fiber over a circle. More precisely, by [21, Thm.1.39], we have the following:

Lemma 2.26. Let $X$ be a CW complex of finite homotopy type, and $f: X \rightarrow S^{1}$ a fibration with connected fiber $F$ of finite homotopy type. Assume that the epimorphism $f_{*}: \pi_{1}(X) \rightarrow \pi_{1}\left(S^{1}\right)=$ $\mathbb{Z}$ admits a factorization $\pi_{1}(X) \stackrel{\alpha}{\rightarrow} \Gamma \stackrel{\beta}{\rightarrow} \mathbb{Z}$, with $\alpha$ and $\beta$ epimorphisms. Then

$$
b_{i}^{(2)}(X, \alpha)=0, \text { for all } i \geq 0 .
$$

Definition 2.27. An epimorphism $\alpha: \pi_{1}(X) \rightarrow \Gamma$ is called $\xi$-admissible if $\xi$ factors through $\alpha$.

We can now prove the following vanishing result for the $L^{2}$-Betti numbers of a space $X$ satisfying the assumptions of Proposition 2.8 (compare also with [23] for a more concrete situation). Recall that for a homomorphism $\xi: \pi_{1}(X) \rightarrow \mathbb{Z}$ (regarded as an element in $H^{1}(X, \mathbb{Z})$ ), we have fixed a homotopy representative $f_{\xi}: X \rightarrow S^{1}$.

Theorem 2.28. Let $X$ be a finite connected CW complex, and fix an epimorphism $\xi: \pi_{1}(X) \rightarrow \mathbb{Z}$ with a homotopy representative $f_{\xi}: X \rightarrow S^{1}$. Assume that $X$ is obtained from a CW (sub-)complex $Z$, by attaching cells of dimension $\geq n$ (with $n \geq 2$ ). Moreover, assume that $\left.f_{\xi}\right|_{Z}: Z \rightarrow S^{1}$ is a locally trivial topological fibration with fiber $F$ of finite homotopy type.. Then, for any $\xi$-admissible epimorphism $\alpha: \pi_{1}(X) \rightarrow \Gamma$, we have that $b_{i}^{(2)}(X, \alpha)=0$ for all $i \leq n-1$. If, moreover, $X$ is obtained from $Z$ by attaching cells of dimension exactly $n$, then $b_{i}^{(2)}(X, \alpha)=0$ for all $i \neq n$, and $b_{n}^{(2)}(X, \alpha)=(-1)^{n} \chi(X)$.

Proof. Since, as shown in the proof of Proposition 2.4, $j_{*}: \pi_{1}(Z) \rightarrow \pi_{1}(X)$ is onto, it follows that $\left.f_{\xi}\right|_{Z}$ induces an epimorphism on fundamental groups, so its fiber is connected. It also follows that $\alpha_{Z}: \pi_{1}(Z) \rightarrow \Gamma$ is an epimorphism, which is clearly admissible for $\xi \circ j_{*}=\left(\left.f_{\xi}\right|_{Z}\right)_{*}$. By Lemma 2.26 applied to the pair $\left(Z, \alpha_{Z}\right)$, we have that $b_{i}^{(2)}\left(Z, \alpha_{Z}\right)=0$ for all $i \geq 0$. The desired result follows now from Proposition 2.25, together with Lemma 2.23(c).

Remark 2.29. The above result proves Corollary 1.9 from the Introduction (see also Remark 4.15)), and gives another topological interpretation of the signed Euler characteristic property (11) for smooth closed subvarieties of semi-abelian varieties. (The proof of Corollary 1.9 is trivial for $\operatorname{dim}(X)=1$, since $X$ is homotopy equivalent to a 1-dimensional CW-complex in this case.) 


\section{NON-PROPER MORSE THEORY}

In this section, we recall the main aspects of the non-proper Morse theory due to Palais-Smale [26], as well as its circle-valued extension. Non-proper Morse theory is the main tool needed in this paper to study the topology of subvarieties of semi-abelian varieties.

3.1. Non-proper Morse theory after Palais-Smale. Let $f: M \rightarrow \mathbb{R}$ be a real-valued smooth function on a smooth manifold $M$. For any real numbers $a<b$, we define $f^{a, b}:=f^{-1}([a, b])$ and $f^{a}:=f^{-1}((-\infty, a])$. The following non-proper Morse theory result is due to Palais-Smale:

Theorem 3.1 ([26]). Let $M$ be a complete Riemannian manifold, and let $f: M \rightarrow \mathbb{R}$ be a real-valued Morse function satisfying the following:

Condition: If $S$ is a subset of $M$ on which $|f|$ is bounded but on which $\|\nabla f\|$ is not bounded away from zero, then there exists a critical point of $f$ in the closure of $S$.

Then the following properties hold:

(1) For any real numbers $a<b$, there are finitely many critical points of $f$ in $f^{a, b}$.

(2) Let $a, b$ be regular values of $f$. Suppose that there are $r$ critical points of $f$ in $f^{a, b}$ having index $d_{1}, \ldots, d_{r}$, respectively. Then $f^{b}$ has the homotopy type of $f^{a}$ with $r$ cells of dimensions $d_{1}, \ldots, d_{r}$ attached.

(3) If $c$ is a regular value of $f$, then $f$ has the structure of a fiber bundle in a small neighborhood of $c$.

Proof. Let us first discuss (3). Let $\epsilon>0$ be sufficiently small such that $f^{-1}([c-\epsilon, c+\epsilon])$ does not contain any critical point of $f$. By the condition on $f,\|\nabla f\|$ is bounded away from zero. Using a partition of unity, we can construct a smooth function on $M$ which is equal to $\|\nabla f\|$ on $f^{-1}([c-\epsilon, c+\epsilon])$ and is bounded away from zero. Dividing the Riemannian metric on $M$ by the above function, we have a new complete Riemannian metric, since the function is bounded away from zero. Moreover, with respect to the new metric, $\|\nabla f\|=1$ on $f^{-1}([c-\epsilon, c+\epsilon])$. Therefore, the gradient flow provides a trivialization of $f$ over $[c-\epsilon, c+\epsilon]$. Thus, $f$ has the structure of a fiber bundle over $(c-\epsilon, c+\epsilon)$.

Properties (1) and (2) are stated without proof in [26, Theorem 2, Page 166]. Nevertheless, they follow from standard Morse theory arguments. For instance, it is not hard to give a proof using the above reasoning together with the standard proper Morse theory.

3.2. Circle-valued non-proper Morse theory. In this section we present a circle-valued version of Theorem 3.1 .

Let $f: M \rightarrow S^{1}$ be a circle-valued smooth function on a smooth manifold. Here we consider

$S^{1}=\mathbb{R} / \mathbb{Z}$. For any real numbers $a, b$ with $a<b<a+1$, we define $f^{\bar{a}, \bar{b}}:=f^{-1}(\overline{[a, b]})$, where $\overline{[a, b]}$ is the image of the closed interval $[a, b]$ in $S^{1}$.

Theorem 3.2. Let $M$ be a complete Riemannian manifold, and let $f: M \rightarrow S^{1}$ be a circle-valued Morse function satisfying the following:

Condition: If $S$ is a subset of $M$ on which $\|\nabla f\|$ is not bounded away from zero, then there exists a critical point of $f$ in the closure of $S$.

Then the following hold:

(1) There exists finitely many critical points of $f$ in $M$; 
(2) Let $a, b$ be real numbers with $a<b<a+1$ such that $\bar{a}$ and $\bar{b}$ are regular values of $f$. Suppose that there are $r$ critical points of $f$ in $f^{\bar{a}, \bar{b}}$ of index $d_{1}, \ldots, d_{r}$, respectively. Then $f^{\bar{a}, \bar{b}}$ has the homotopy type of $f^{-1}(\bar{a})$ with $r$ cells of dimensions $d_{1}, \ldots, d_{r}$ attached.

(3) If $\bar{c}$ is a regular value of $f$, then $f$ has the structure of a fiber bundle in a small neighborhood of $\bar{c}$.

Proof. Let $\tilde{f}: \widetilde{M} \rightarrow \mathbb{R}$ be the lifting of $f: M \rightarrow S^{1}$ to the infinite cyclic cover $\widetilde{M}$ corresponding to $f$. Then by lifting $S$ to the fundamental domain $\tilde{f}^{-1}([a, a+1))$, one can easily check that the Palais-Smale condition of Theorem 3.1 applies to $\tilde{f}$. Thus (1) and (3) follow from the corresponding statements of Theorem 3.1 .

By Theorem 3.1 (3), there is a diffeomorphism between $\tilde{f}^{-1}((a-\epsilon, a))$ and $\tilde{f}^{-1}(a) \times(a-\epsilon, a)$ for some small $\epsilon>0$. Thus, we can glue $\tilde{f}^{-1}(a-\epsilon,+\infty)$ with $\tilde{f}^{-1}(a) \times(-\infty, a)$ via the above diffeomorphism and obtain a new manifold $\widetilde{M^{\prime}}$ and a function $\widetilde{f}^{\prime}: \widetilde{M^{\prime}} \rightarrow \mathbb{R}$. We can construct a complete Riemannian metric on $\widetilde{M}^{\prime}$ as follows. Let $\rho: \mathbb{R} \rightarrow \mathbb{R}$ be a smooth non-decreasing function, such that $\rho=0$ on $(-\infty, a-\epsilon]$ and $\rho=1$ on $[a,+\infty)$. Define the metric on $\widetilde{M}^{\prime}$ to be

$$
g_{\widetilde{M}^{\prime}}=(1-\rho) \cdot g_{\widetilde{f}^{-1}(a) \times(-\infty, a)}+\rho \cdot g_{\widetilde{f}^{-1}(a-\epsilon,+\infty)},
$$

which is clearly complete. Now, notice that $\left(\tilde{f}^{\prime}\right)^{b}$ is homotopy equivalent to $f^{\bar{a}, \bar{b}}$ and $\left(\tilde{f}^{\prime}\right)^{a}$ is homotopy equivalent to $f^{-1}(\bar{a})$. Thus (2) follows from Theorem 3.1 (2) applied to $\left(\widetilde{M}^{\prime}, \widetilde{f}^{\prime}\right)$.

\section{Topology of Semi-ABelian varieties. Statements of Results}

In this section, we introduce the main constructions and results of this note. We defer some of the technical proofs for subsequent sections. We also prove Theorem 1.1 (see Corollary 4.7) and Theorem 1.2 (see Corollary 4.14 and Corollary 4.17).

4.1. Subvarieties of semi-abelian varieties. A nice introduction of complex semi-abelian varieties is [25, Chapter5]. We recall here the following definition.

Definition 4.1. A commutative complex algebraic group $G$ is called a semi-abelian variety if there is a short exact sequence of complex algebraic groups

$$
1 \rightarrow T \rightarrow G \rightarrow A \rightarrow 1
$$

where $T$ is an affine torus and $A$ is an abelian variety.

Let $X$ be an $n$-dimensional smooth connected closed subvariety of a semi-abelian variety $G$. Without loss of generality, we will assume that $X$ contains the origin $e$ of $G$, and we will always take $e$ as the base point of $G$ and $X$, respectively. Let $\Gamma$ be the space of left invariant holomorphic 1 -forms on $G$. For a left invariant 1 -form $\eta \in \Gamma$, we denote its restriction to $X$ by $\eta_{X}$. Suppose the class $[\operatorname{Re}(\eta)] \in H^{1}(G ; \mathbb{R})$ is integral, i.e., $[\operatorname{Re}(\eta)]$ is in the image of the natural map $H^{1}(G ; \mathbb{Z}) \rightarrow$ $H^{1}(G ; \mathbb{R})$. Then $\left[\operatorname{Re}\left(\eta_{X}\right)\right] \in H^{1}(X ; \mathbb{R})$ is also integral. The map

$$
x \mapsto \int_{e}^{x} \operatorname{Re}\left(\eta_{X}\right)
$$

given by integration along paths defines a multi-valued map from $X$ to $\mathbb{R}$. Since the ambiguity lies in the subgroup $\mathbb{Z} \subset \mathbb{R}$, we have a well-defined smooth map

$$
\int \operatorname{Re}\left(\eta_{X}\right): X \rightarrow S^{1}
$$


which we denote by $\Phi_{\eta}$. Notice that $\Phi_{\eta}$ is the restriction of $\int \operatorname{Re}(\eta): G \rightarrow S^{1}$, which we denote by $\Phi_{\eta, G}$. Moreover, $\Phi_{\eta, G}$ is a homomorphism of real Lie groups, because $\operatorname{Re}(\eta)$ is a left invariant 1-form on $G$.

In the above notations we have the following result, whose proof will be given in Section 6 .

Theorem 4.2. Regarding $\Gamma$ as a complex affine space, there exists a non-empty Zariski subset $U$ of $\Gamma$ such that the following holds: if $\eta \in U$ with $[\operatorname{Re}(\eta)] \in H^{1}(G ; \mathbb{R})$ integral, then $\Phi_{\eta}: X \rightarrow S^{1}$ is a circle-valued Morse function which satisfies the the circle-valued Palais-Smale condition of Theorem 3.2 .

Remark 4.3. The metric on $X$ is the restriction of any left invariant Riemannian metric on $G$. Since any left invariant Riemannian metric on a Lie group $G$ is complete, and any closed submanifold of a complete Riemannian manifold is also complete, it follows that $X$ is a complete Riemannian manifold. Furthermore, since any two left invariant Riemannian metrics $g_{1}$ and $g_{2}$ on $G$ are equivalent, i.e., $\frac{1}{M} g_{1} \leq g_{2} \leq M g_{1}$ for sufficiently large $M$, the statement of Theorem 4.2 does not depend on the choice of the left invariant metric on $G$.

Corollary 4.4. Suppose $\eta \in U$ is chosen as in the Theorem 4.2 and assume $[\operatorname{Re}(\eta)] \in H^{1}(G ; \mathbb{R})$ is integral. Let $\widetilde{X}$ be the infinite cyclic cover of $X$ with respect to the map $\Phi_{\eta}: X \rightarrow S^{1}$, and let $\widetilde{\Phi}_{\eta}: \widetilde{X} \rightarrow \mathbb{R}$ be the lifting of $\Phi_{\eta}: X \rightarrow S^{1}$. Then for a regular value a of $\widetilde{\Phi}_{\eta}, \widetilde{X}$ has the homotopy type of $\widetilde{\Phi}_{\eta}^{-1}(a)$ with possibly infinitely many $n$-cells attached.

Proof. Since $\eta$ is a holomorphic 1-form, locally the Morse function $\Phi_{\eta}$ is equal to the real part of a holomorphic Morse function. By the holomorphic Morse lemma (see, e.g., [29, Section 2.1.2]), the index of $\Phi_{\eta}$ at every critical point is equal to the complex dimension of $X$. The assertion follows now from Theorem 4.2 and Theorem 3.2 .

In order to apply the topological vanishing results to the semi-abelian setting, we need $\widetilde{\Phi}_{\eta}^{-1}(a)$ to have finite homological or finite homotopy type. This is always the case when $G$ is an abelian variety, because then $\widetilde{\Phi}_{\eta}^{-1}(a)$ is a compact real analytic space. However, when $G$ is not compact, $\widetilde{\Phi}_{\eta}^{-1}(a)$ may not have finite homotopy type. For example, when $X=G=\mathbb{C}^{*}$ with coordinate $z$, and $\eta=2 \pi \sqrt{-1} d z / z, \Phi_{\eta}: \mathbb{C}^{*} \rightarrow S^{1}$ is the composition of the following two maps

$$
\mathbb{C}^{*} \rightarrow \mathbb{R} \rightarrow S^{1}
$$

where the first map is defined by $z \mapsto \log |z|$ and the second is taking the quotient by $\mathbb{Z}$. Clearly, the fibers of $\Phi_{\eta}: \mathbb{C}^{*} \rightarrow S^{1}$ have infinitely many connected components, so the same holds for $\widetilde{\Phi}_{\eta}$.

Let $T$ be the affine torus in the semi-abelian variety $G$ such that the quotient $G / T$ is an abelian variety. Denote the restriction of $\eta$ to $T$ by $\eta_{T}$. We have the following result whose proof will be given in Section 5 .

Theorem 4.5. Let $\Phi_{\eta}: X \rightarrow S^{1}$ be the map defined by $\eta \in \Gamma$ (not necessarily in $U$ ) with $[\operatorname{Re}(\eta)] \in H^{1}(G ; \mathbb{R})$ integral. If, furthermore, the cohomology class $\left[\eta_{T}\right] \in H^{1}(T ; \mathbb{C})$ is real, i.e., in the image of $H^{1}(T ; \mathbb{R}) \rightarrow H^{1}(T ; \mathbb{C})$, then for any $\bar{a} \in S^{1}, \Phi_{\eta}^{-1}(\bar{a})$ has finite homotopy type.

When $\operatorname{dim} T>0$, the real linear map

$$
\Gamma \longrightarrow H^{1}(G ; \mathbb{R})
$$

given by $\eta \mapsto[\operatorname{Re}(\eta)]$ is surjective but not injective. In other words, given a group homomorphism $\xi: \pi_{1}(G) \rightarrow \mathbb{Z}$, there may exist many $\eta \in \Gamma$ such that the homomorphism induced by $\Phi_{\eta}: G \rightarrow S^{1}$ 
on fundamental groups coincides with $\xi$. However, if we restrict the above map to $\left\{\eta \in \Gamma \mid\left[\eta_{T}\right] \in\right.$ $H^{1}(T ; \mathbb{C})$ is real $\}$, then such $\eta$ is unique. More precisely, we have the following result, whose proof will be given in Section 5 .

Proposition 4.6. For any class $a \in H^{1}(G ; \mathbb{R})$, there exists a unique $\eta \in \Gamma$ such that $[\operatorname{Re}(\eta)]=a \in$ $H^{1}(G ; \mathbb{R})$ and $\left[\eta_{T}\right] \in H^{1}(T ; \mathbb{C})$ is real, i.e., in the image of the natural map $H^{1}(T ; \mathbb{R}) \rightarrow H^{1}(T ; \mathbb{C})$.

Corollary 4.7. Let $X$ be an $n$-dimensional closed connected smooth subvariety of a complex semiabelian variety $G$. For a generic group homomorphism $\xi: \pi_{1}(X) \rightarrow \mathbb{Z}$, the corresponding infinite cyclic cover $X^{\xi}$ is homotopy equivalent to a finite $C W$-complex with possibly infinitely many $n$-cells attached. Moreover, if $\xi$ is surjective, then $X^{\xi}$ is connected.

Remark 4.8. The meaning of "generic" in the above result is with respect to some Zariski topology on $\operatorname{Hom}\left(\pi_{1}(X), \mathbb{Z}\right)$. In the proof below, we will construct a complex vector space $\Gamma^{\prime}$ and an injective map $\operatorname{Hom}\left(\pi_{1}(X), \mathbb{Z}\right) \rightarrow \Gamma^{\prime}$. There is a non-empty Zariski open subset $U^{\prime}$ of $\Gamma^{\prime}$ such that the statement holds for all $\xi: \pi_{1}(X) \rightarrow \mathbb{Z}$ whose image in $\Gamma^{\prime}$ is contained in $U^{\prime}$.

Proof. Let $a_{X}: X \rightarrow A l b(X)$ be the generalized Albanese map. By the universal property of the generalized Albanese map, the closed embedding $X \hookrightarrow G$ factors as $X \stackrel{a_{X}}{\longrightarrow} A l b(X) \rightarrow G$. Since the composition $X \rightarrow G$ is a closed embedding, the first map $a_{X}: X \rightarrow A l b(X)$ must also be a closed embedding. Denote $A l b(X)$ by $G^{\prime}$ and consider $X$ as a subvariety of $G^{\prime}$ via the Albanese map.

By definition, $\left(a_{X}\right)_{*}: H_{1}(X ; \mathbb{Z}) \rightarrow H_{1}\left(G^{\prime} ; \mathbb{Z}\right)$ is surjective, and its kernel is equal to the torsion subgroup of $H_{1}(X ; \mathbb{Z})$. Thus, there is a canonical bijection between $\operatorname{Hom}\left(\pi_{1}(X), \mathbb{Z}\right)$ and $\operatorname{Hom}\left(\pi_{1}\left(G^{\prime}\right), \mathbb{Z}\right)$. Since $H_{1}(X ; \mathbb{Z})$ is the abelianization of $\pi_{1}(X)$, and by the universal coefficient theorem, we have

$$
\operatorname{Hom}\left(\pi_{1}\left(G^{\prime}\right), \mathbb{Z}\right) \cong \operatorname{Hom}\left(H_{1}\left(G^{\prime} ; \mathbb{Z}\right), \mathbb{Z}\right) \cong H^{1}\left(G^{\prime} ; \mathbb{Z}\right) .
$$

Let $\Gamma^{\prime}$ be the space of left invariant holomorphic 1-forms on $G^{\prime}$. By Proposition 4.6, there is a natural map $H^{1}\left(G^{\prime} ; \mathbb{Z}\right) \rightarrow \Gamma^{\prime}$ defined by $a \mapsto \eta$. Composing the maps $\operatorname{Hom}\left(\pi_{1}(X), \mathbb{Z}\right) \rightarrow$ $\operatorname{Hom}\left(\pi_{1}\left(G^{\prime}\right), \mathbb{Z}\right), \operatorname{Hom}\left(\pi_{1}\left(G^{\prime}\right), \mathbb{Z}\right) \cong H^{1}\left(G^{\prime} ; \mathbb{Z}\right)$, and, resp., $H^{1}\left(G^{\prime} ; \mathbb{Z}\right) \rightarrow \Gamma^{\prime}$, we obtain a map $\operatorname{Hom}\left(\pi_{1}(X), \mathbb{Z}\right) \rightarrow \Gamma^{\prime}$, which induces an isomorphism between $\operatorname{Hom}\left(\pi_{1}(X), \mathbb{Z}\right)$ and a discrete subgroup of $\Gamma^{\prime}$.

Let $U^{\prime}$ be the subset of $\Gamma^{\prime}$ chosen as in Theorem 4.2. Suppose the element of $\Gamma^{\prime}$ corresponding to $\xi: \pi_{1}(X) \rightarrow \mathbb{Z}$ is contained in $U^{\prime}$. By Theorem 4.2. Theorem 3.2 and Theorem 4.5, it follows that $X^{\xi}$ is homotopy equivalent to a finite CW-complex with possibly infinitely many $n$-cells attached.

One can easily see that $\pi_{0}\left(X^{\xi}\right)$ is isomorphic to the cokernel of $\xi: \pi_{1}(X) \rightarrow \mathbb{Z}$. Thus, if $\xi$ is surjective, then $X^{\xi}$ is connected.

Remark 4.9. In the preceding corollary, the Zariski topology on $\operatorname{Hom}\left(\pi_{1}(X), \mathbb{Z}\right)$ is coarser than the standard Zariski topology on $\operatorname{Hom}\left(\pi_{1}(X), \mathbb{Z}\right) \cong \mathbb{Z}^{b_{1}(X)}$. Evidently, the subset of surjective maps in $\operatorname{Hom}\left(\pi_{1}(X), \mathbb{Z}\right)$ is dense with respect to the standard Zariski topology on $\operatorname{Hom}\left(\pi_{1}(X), \mathbb{Z}\right) \cong \mathbb{Z}^{b_{1}(X)}$. Thus, the set

$$
\left\{\xi \in \operatorname{Hom}\left(\pi_{1}(X), \mathbb{Z}\right) \mid \xi \text { is surjective and generic }\right\}
$$

is non-empty. 
4.2. Subvarieties of affine torus. In the case when $G$ is an affine torus, the topology of smooth subvarieties of $G$ can be made even more explicit.

Under the notations of Theorem 4.2, suppose moreover that $G$ is an affine torus. Let $\eta \in U$ such that $[\eta] \in H^{1}(G ; \mathbb{C})$ is integral, i.e., in the image of the natural map $H^{1}(G ; \mathbb{Z}) \rightarrow H^{1}(G ; \mathbb{C})$. Via the isomorphism $\mathbb{C} / \mathbb{Z} \cong \mathbb{C}^{*}$, the integration along paths starting at $e$

$$
x \mapsto \int_{e}^{x} \eta
$$

defines a map $\int \eta: G \rightarrow \mathbb{C}^{*}$, which is a homomorphism of algebraic groups. Denote the composition of $\int \eta$ and $\log |\cdot|: \mathbb{C}^{*} \rightarrow \mathbb{R}$ by $\Psi_{\eta, G}:=\log \left|\int \eta\right|: G \rightarrow \mathbb{R}$. Let $\Psi_{\eta}$ be the restriction of $\Psi_{\eta, G}$ to $X$. The following result will be proved in Section 6 .

Theorem 4.10. Suppose $\eta \in U$ is chosen as in the Theorem 4.2 and assume $[\eta] \in H^{1}(G ; \mathbb{C})$ is integral. Then the function $\Psi_{\eta}: X \rightarrow \mathbb{R}$ is a Morse function which satisfies the the Palais-Smale condition of Theorem 3.1 .

Corollary 4.11. Suppose $\eta \in U$ is chosen as in the Theorem [4.2 and assume $[\eta] \in H^{1}(G ; \mathbb{C})$ is integral. Then for a sufficiently negative real number $a, \Psi_{\eta}^{-1}((-\infty, a])$ is a fiber bundle over $S^{1}$, whose fiber is homotopy equivalent to a finite $C W$-complex. Moreover, $X$ has the homotopy type of $\Psi_{\eta}^{-1}((-\infty, a])$ with finitely many $n$-cells attached.

Proof. The map $\int \eta_{X}: X \rightarrow \mathbb{C}^{*}$ is a morphism of smooth complex algebraic varieties. Hence, by the generic fibration theorem (see [28, Corollary 5.1]), for sufficiently small $\epsilon>0$, the map $\int \eta_{X}$ is a fiber bundle over the punctured disc $D_{\epsilon}(0) \backslash\{0\}$, whose fibers are algebraic varieties. Thus, the first part of the corollary follows.

As a morphism of algebraic varieties, $\int \eta_{X}: X \rightarrow \mathbb{C}^{*}$ has only finitely many critical values. The critical values of $\Psi_{\eta}$ are the logarithms of the absolute values of the critical values of $\int \eta_{X}$. Hence $\Psi_{\eta}$ has only finitely many critical values. In the proof of Theorem 4.10, we will show that $\int \eta_{X}: X \rightarrow \mathbb{C}^{*}$ is a holomorphic Morse function. A standard fact is that the absolute value of a non-vanishing holomorphic Morse function on a complex manifold of dimension $n$ is a real Morse function whose index at each critical point is $n$ (e.g., the argument in [19][Lemma 2.1] can be easily adapted to the general case). Since $\log : \mathbb{R}_{>0} \rightarrow \mathbb{R}$ is a smooth function with positive derivatives, $\Psi_{\eta}: X \rightarrow \mathbb{R}$ is a real-valued Morse function, which has only finitely many critical points, all of which are of index $n$. Thus, the second part of the corollary follows from Theorem 4.10 and Theorem 3.1 .

Remark 4.12. The fiber in the above statement is connected if the integral cohomology class $\eta$ is not divisible by any integer $>1$. (Indeed, under this assumption, the homomorphism induced by the bundle map on fundamental groups is onto, so the fiber is connected.) As in Remark 4.9, it can be seen that there exists $\eta \in U$ so that $[\eta]$ (when regarded as an integral class) is not divisible by any integer $>1$.

Remark 4.13. Obviously, the number of $n$-cells attached in the the statement of Corollary 4.11 is equal to the number of critical points of $\Psi_{\eta}$. It follows from the arguments in the proof that the critical points of $\Psi_{\eta}$ are same as the degeneration points of $\eta_{X}$. Thus, the number of $n$-cells attached is equal to the number of degeneration points of $\eta_{X}$.

Corollary 4.14. Let $G$ be an affine torus, and let $X \subseteq G$ be a smooth connected closed subvariety of dimension $n$. Then for any generic homomorphism $\xi: \pi_{1}(X) \rightarrow \mathbb{Z}$, which factors through 
$\pi_{1}(X) \rightarrow \pi_{1}(G)$, there exists a continuous map $F_{\xi}: X_{0} \rightarrow S^{1}$ defined on a subset $X_{0}$ of $X$ such that the following properties hold:

(1) $F_{\xi}$ is a fibration whose fiber is of finite homotopy type.

(2) $X$ is homotopy equivalent to $X_{0}$ with finitely many $n$-cells attached.

Proof. The meaning of "generic" in the above statement is with respect to the standard Zariski topology on $\operatorname{Hom}\left(G, \mathbb{C}^{*}\right) \cong \mathbb{Z}^{\operatorname{dim} G}$.

On the affine torus $G$, the map $\eta \mapsto[\eta]: \Gamma \rightarrow H^{1}(G ; \mathbb{C})$ is an isomorphism. Thus, each class $H^{1}(G ; \mathbb{Z})$ is represented by a unique left invariant holomorphic 1 -form. By (2), each homomorphism $\xi: \pi_{1}(X) \rightarrow \mathbb{Z}$ corresponds to a unique integral cohomology class in $H^{1}(X, \mathbb{Z})$. Choose $\xi$ such that the corresponding cohomology class is $\eta_{X}$, where $\eta$ is generic as in Theorem 4.2. Set $X_{0}=\Psi_{\eta}^{-1}((-\infty, a])$ for sufficiently negative $a$, and let $F_{\xi}$ be the restriction of the composition $X \stackrel{\int \eta}{\longrightarrow} \mathbb{C}^{*} \stackrel{\arg }{\longrightarrow} S^{1}$ to $X_{0}$. The assertion follows now from the Corollary 4.11.

Remark 4.15. Note that if $\xi$ in Corollary 4.14 is an epimorphism and $n>1$, the fiber of the fibration $F_{\xi}$ is connected. Indeed, as in the proof of Proposition 2.4 the homomorphism $\pi_{1}\left(X_{0}\right) \rightarrow \pi_{1}(X)$ induced by inclusion is in this case onto, so $F_{\xi}$ induces an epimorphism on fundamental groups. When $n=1, X$ is homotopy equivalent to a connected 1-dimensional CW complex (since it is affine).

Remark 4.16. In the case of a very affine manifold $X \subseteq G$ as in the above corollary, the homomorphism $\pi_{1}(X) \rightarrow \pi_{1}(G)$ induced by inclusion is non-trivial, provided that $\operatorname{dim} X>0$. In more detail, let $h$ be the restriction to $X$ of the polynomial map $\prod_{i=1}^{m} z_{i}: G \rightarrow \mathbb{C}^{*}$, where $\left(z_{1}, \cdots, z_{m}\right)$ are the coordinates of $G$. Without any loss of generality, we may assume that $h$ is dominant. (If not, the image of $h$ is just one point, say $b \in \mathbb{C}^{*}$, since $X$ is connected. Then $G_{b}:=\left\{\prod_{i=1}^{m} z_{i}=b\right\}$ is a complex subtorus of $G$, of complex codimension one, which contains $X$. Replace $G$ by $G_{b}$. After finitely steps of replacing the torus containing $X$ by codimension-one subtori, the map $h$ becomes dominant.) Then it is easy to see that the homomorphism $h_{*}: \pi_{1}(X) \rightarrow \pi_{1}\left(\mathbb{C}^{*}\right)$ induced by $h$ on fundamental groups is non-trivial, provided that $\operatorname{dim} X>0$. Since, by definition, $h_{*}$ factors trough $\pi_{1}(X) \rightarrow \pi_{1}(G)$, it follows that the latter is also a non-trivial homomorphism.

A more intrinsic version of the above Corollary 4.14 can be given as follows.

Corollary 4.17. Let $X$ be an n-dimensional smooth connected closed subvariety of an affine torus $G$. Suppose the mixed Hodge structure of $H^{1}(X ; \mathbb{Z})$ is pure of type $(1,1)$, or equivalently, there exists a smooth compactification $\bar{X}$ of $X$ such that $b_{1}(\bar{X})=0$. Then for a generic homomorphism $\xi: \pi_{1}(X) \rightarrow \mathbb{Z}$, there exists a continuous map $F_{\xi}: X_{0} \rightarrow S^{1}$ defined on a subset $X_{0}$ of $X$ such that the following properties hold:

(1) $F_{\xi}$ is a fibration whose fiber is of finite homotopy type.

(2) $X$ is homotopy equivalent to $X_{0}$ with finitely many $n$-cells attached.

Proof. Note that $\operatorname{Hom}\left(\pi_{1}(X), \mathbb{Z}\right)$ is a free abelian group of rank $b_{1}(X)$. The meaning of "generic" in the above statement is with respect to the standard Zariski topology on $\operatorname{Hom}\left(\pi_{1}(X), \mathbb{Z}\right) \cong \mathbb{Z}^{b_{1}(X)}$.

Let $a_{X}: X \rightarrow A l b(X)$ be the generalized Albanese map of $X$. Since $H^{1}(X ; \mathbb{Z})$ is of type $(1,1)$, $A l b(X)$ is an affine torus. By the same argument as in the proof of Corollary 4.7 the Albanese map $a_{X}: X \rightarrow A l b(X)$ is a closed embedding. Then the conclusion follows from the Corollary 4.14 with $G$ replaced by $A l b(X)$. 


\subsection{Proof of Theorem 1.11.}

Proof of Theorem 1.11. The statements (i) and (ii) follow easily from the arguments in the beginning of Section 6

To prove (iii), let us first assume that $G$ is an affine torus and $[\operatorname{Re}(\eta)] \in H^{1}(G ; \mathbb{R})$ is integral. Then (iii) follows from Proposition 2.10, Corollary 4.11] and Remark 4.13. Notice that the number of critical points is constant on a non-empty Zariski open set of $\Gamma$ (see the arguments in the beginning of Section [6). Since the subset $\left\{\eta \in \Gamma \mid[\operatorname{Re}(\eta)] \in H^{1}(G ; \mathbb{R})\right.$ is integral $\}$ is clearly Zariski dense in $\Gamma$, the statement (iii) follows assuming $G$ is an affine torus.

When $G$ is a general semi-abelian variety, we can apply a similar argument, but using Corollary 4.7 and Theorem 4.5 instead of Corollary 4.11, while instead of Remark 4.13 we need the parallel fact that the number of critical points of $\Psi_{\eta}$ is equal to the number of degeneration points of $\eta$.

Indeed, it follows from Corollary 4.4 that for a regular value $a \in \mathbb{R}$ of $\widetilde{\Phi}_{\eta}$, the infinite cyclic cover $\widetilde{X}$ corresponding to $\Phi_{\eta}$ is homotopy equivalent to $\widetilde{\Phi}_{\eta}^{-1}(a)$ by adding $n$-cells corresponding to the degeneration points of $\eta_{X}$. Moreover, we have an isomorphism of $\mathbb{C}\left[t, t^{-1}\right]$-modules:

$$
H_{n}\left(\widetilde{X}, \widetilde{\Phi}_{\eta}^{-1}(a) ; \mathbb{C}\right) \cong \mathbb{C}\left[t, t^{-1}\right]^{\oplus \ell},
$$

where $\ell$ is the number of degeneration points of $\eta_{X}$. We also have that $H_{i}\left(\widetilde{X}, \widetilde{\Phi}_{\eta}^{-1}(a) ; \mathbb{C}\right) \cong 0$, for any $i \neq n$.

Assume now that $\eta_{X} \in H^{1}(X ; \mathbb{R})$ is integral. Then the assumptions of Theorem 4.5 are automatically satisfied, hence $\widetilde{\Phi}_{\eta}^{-1}(a)$ has finite homotopy type. Let $\xi$ denote the homomorphism $\xi: \pi_{1}(X) \rightarrow \mathbb{Z}$ corresponding to $\eta_{X}$. Then the corresponding infinite cyclic cover $X^{\xi}$ coincides with $\widetilde{X}$. By (9) and from the arguments in the proof of Proposition 2.10 , we get that $H_{i}\left(X^{\xi} ; \mathbb{C}\right)$ is a torsion $\mathbb{C}\left[t, t^{-1}\right]$-module for $i \neq n$, and

$$
\ell=\operatorname{rank}_{\mathbb{C}\left[t, t^{-1}\right]} H_{n}\left(X^{\xi} ; \mathbb{C}\right)=(-1)^{n} \chi(X) .
$$

The case when $\eta_{X} \in H^{1}(X ; \mathbb{C})$ is non-integral follows from the same argument as in the affine torus case.

Remark 4.18. The statement (iii) of Theorem 1.11 follows essentially from [13]. One can also replace the index theorem of [13] by the Chern class argument in [17 to conclude statement (iii). Nevertheless, both proofs require some sophisticated forms of intersection theory extended to the non-compact setting. In contrast, our proof only uses Morse theory. Especially when $G$ is an affine torus, our proof does not rely on a compactification of $G$ or $X$, and is much more elementary.

\section{Semi-abelian varieites. Proof of structure Results}

In this section, we review the definition of complex semi-abelian varieties and discuss some compactifications as complex and real algebraic varieties. We will prove Theorem 4.5 using a compactification of $G_{\mathbb{R}}$, the underlying real algebraic variety underlying $G$. We also supply here a proof of Proposition 4.6.

Recall that a commutative complex algebraic group $G$ is called a semi-abelian variety if there is a short exact sequence of complex algebraic groups

$$
1 \rightarrow T \rightarrow G \rightarrow A \rightarrow 1
$$


where $T$ is an affine torus and $A$ is an abelian variety. Let $m=\operatorname{dim} T$. As a complex Lie group, an $N$-dimensional semi-abelian variety $G$ is isomorphic to $\mathbb{C}^{N} / \Lambda$, where $\Lambda \subset \mathbb{C}^{N}$ is a discrete subgroup generating $\mathbb{C}^{N}$ as a complex vector space.

Even though the following result is not directly applicable to the proof of Theorem 4.5, it motivates our later constructions.

Lemma 5.1 ([13]). There exists a projective compactification $\bar{G}$ of $G$, such that $\bar{G}$ is a $\mathbb{C P}^{m}$-bundle over $A$, which is constructed as a fiberwise $\mathbb{C P}^{m}$-compactification of $T \cong\left(\mathbb{C}^{*}\right)^{m}$.

Proof. By choosing a splitting $T \cong \mathbb{C}^{*} \times \cdots \times \mathbb{C}^{*} \cong\left(\mathbb{C}^{*}\right)^{m}$, we can write $G$ as

$$
G=G_{1} \times_{A} G_{2} \times_{A} \cdots \times_{A} G_{m}
$$

where each $G_{k}$ is an extension of $A$ by $\mathbb{C}^{*}$. Then each $G_{k}$ is a principal $\mathbb{C}^{*}$-bundle over $A$. Let $L_{k}$ be the flat line bundle on $A$, whose principal $\mathbb{C}^{*}$-bundle is isomorphic to $G_{k}$. In other words, $L_{k}:=\left(G_{k} \times \mathbb{C}\right) / \mathbb{C}^{*}$, where $\mathbb{C}^{*}$ acts on $G_{k}$ by fiberwise multiplication, and $\mathbb{C}^{*}$ acts on $\mathbb{C}$ by scalar multiplication. There is a canonical open embedding $G_{k} \rightarrow L_{k}$, mapping $t \in G_{k}$ to the image of $(t, 1)$ in the quotient $\left(G_{k} \times \mathbb{C}\right) / \mathbb{C}^{*}=L_{k}$.

By construction, each $L_{k}$ is an algebraic vector bundle on $A$. Thus, the total space of $L_{1}+L_{2}+$ $\cdots+L_{m}$ has a natural algebraic variety structure. Let $\bar{G}$ be the fiberwise projective compactification of $L_{1}+L_{2}+\cdots+L_{m}$. In other words, $\bar{G}$ is the associated projective bundle of $L_{1}+L_{2}+\cdots+L_{m}+$ $\mathbb{C}_{A}$, where $\mathbb{C}_{A}$ is the trivial line bundle on $A$. Thus, $\bar{G}$ is a smooth projective variety. Consider $G$ as an open subvariety of $\bar{G}$ by the composition of natural open embeddings

$$
G=G_{1} \times_{A} G_{2} \times_{A} \cdots \times_{A} G_{m} \rightarrow L_{1}+L_{2}+\cdots+L_{m} \rightarrow \bar{G} .
$$

The assertions follow immediately from the above construction.

Since the affine torus $T \cong\left(\mathbb{C}^{*}\right)^{m}$ fits naturally into a short exact sequence of real Lie groups,

$$
1 \rightarrow\left(\mathbb{R}_{>0}\right)^{m} \rightarrow T \rightarrow\left(S^{1}\right)^{m} \rightarrow 1,
$$

and since $A \cong\left(S^{1}\right)^{2 N-2 m}$ as real Lie groups, the short exact sequence (10) induces a short exact sequence

$$
1 \rightarrow \mathbb{R}_{>0}^{m} \rightarrow G_{\mathbb{R}} \stackrel{\sigma}{\rightarrow}\left(S^{1}\right)^{2 N-m} \rightarrow 1
$$

of real Lie groups. In the notations of Section 4, we have the following:

Lemma 5.2. Given $\eta \in \Gamma$, suppose that the class $[\operatorname{Re}(\eta)] \in H^{1}(G ; \mathbb{R})$ is integral. Then the following statements are equivalent:

(i) the class $\left[\eta_{T}\right] \in H^{1}(T, \mathbb{C})$ is integral;

(ii) the class $\left[\eta_{T}\right] \in H^{1}(T, \mathbb{C})$ is real;

(iii) the left invariant holomorphic 1-form $\eta_{T}$ on $T$ is of the form

$$
\eta_{T}=(2 \pi \sqrt{-1})^{-1}\left(n_{1} \frac{d z_{1}}{z_{1}}+\cdots+n_{m} \frac{d z_{m}}{z_{m}}\right)
$$

where $n_{i} \in \mathbb{Z}$, with respect to a choice of coordinates $z_{1}, \ldots, z_{m}$ on $T \cong\left(\mathbb{C}^{*}\right)^{m}$;

(iv) the map $\Phi_{\eta, G}=\int \operatorname{Re}(\eta): G \rightarrow S^{1}$ contracts the Lie subgroup $\left(\mathbb{R}_{>0}\right)^{m}$ of $G$. In other words, $\Phi_{\eta, G}$ factors through $\sigma: G \rightarrow\left(S^{1}\right)^{2 N-m}$. 
Proof. First, we prove (i) $\Longleftrightarrow$ (ii). Since $[\operatorname{Re}(\eta)] \in H^{1}(G ; \mathbb{R})$ is integral, its restriction to $T$, $\left[\operatorname{Re}\left(\eta_{T}\right)\right] \in H^{1}(T ; \mathbb{R})$ is also integral. Thus, the class $\left[\eta_{T}\right] \in H^{1}(T ; \mathbb{C})$ is integral if and only if $\left[\operatorname{Im}\left(\eta_{T}\right)\right]=0 \in H^{1}(T ; \mathbb{R})$.

Since $\int \operatorname{Re}(\eta): G \rightarrow S^{1}$ is a real Lie group homomorphism, (iv) holds if and only if $\int \operatorname{Re}\left(\eta_{T}\right)$ : $T \rightarrow S^{1}$ contracts the Lie subgroup $\left(\mathbb{R}_{>0}\right)^{m}$ of $T$. Both (ii) $\Longleftrightarrow$ (iii) and (iii) $\Longleftrightarrow$ (iv) follow from straightforward computations by using the polar coordinates $\left(r_{i}, \theta_{i}\right)$.

5.1. Proof of Theorem 4.5. We first construct a compactification of $X$, such that the map $\Phi_{\eta}: X \rightarrow S^{1}$ extends to this compactification. Then we can use the stratified Morse theory of Goresky-McPherson to show that the fibers of $\Phi_{\eta}$ have finite homotopy type. Notice that $\Phi_{\eta}: X \rightarrow S^{1}$ is the restriction of $\Phi_{\eta, G}=\int \operatorname{Re}(\eta): G \rightarrow S^{1}$ to $X$. Naturally, we want to construct a compactification of $G$ and let the compactification of $X$ be the closure of $X$ in the compactification of $G$.

Such compactification of $G$ does not exist in the category of smooth complex algebraic varieties. In fact, since the fundamental group is a birational invariant for smooth complex projective varieties, by Lemma [5.1] the fundamental group of any smooth compactification of $G$ is isomorphic to $\pi_{1}(A)$. However, the induced map $\left(\Phi_{\eta}\right)_{*}: \pi_{1}(X) \rightarrow \pi_{1}\left(S^{1}\right)$ may not factor through $\pi_{1}(A)$ in general. Instead, we will construct such a compactification of $G$ as a real algebraic variety.

In this section, we will frequently consider $G$ and $X$ as real algebraic varieties whose dimensions are twice their complex dimensions. To emphasize the real algebraic variety structures, we will denote them by $G_{\mathbb{R}}$ and $X_{\mathbb{R}}$, respectively. We will also use $G_{\mathbb{R}}$ when we consider $G$ as a real Lie group.

Since $\mathbb{R}_{>0}^{m}$ is not a real algebraic group, (11) is not a short exact sequence of real algebraic groups. Notice that $\mathbb{C}^{*}$ with the underlying real algebraic group structure fits into a short exact sequence

$$
1 \rightarrow \mathbb{R}^{*} \rightarrow \mathbb{C}^{*} \rightarrow S^{1} \rightarrow 1
$$

of real algebraic groups, where the first arrow is the natural inclusion, and the second map is given by

$$
x+\sqrt{-1} y \mapsto\left(\frac{x^{2}-y^{2}}{x^{2}+y^{2}}, \frac{2 x y}{x^{2}+y^{2}}\right) .
$$

Thus, the composition $\left(\mathbb{R}^{*}\right)^{m} \rightarrow T_{\mathbb{R}}=\left(\mathbb{C}^{*}\right)^{m} \rightarrow G$ is a homomorphism of real algebraic groups, and we have a short exact sequence of real algebraic groups

$$
1 \rightarrow\left(\mathbb{R}^{*}\right)^{m} \rightarrow G_{\mathbb{R}} \stackrel{\tau}{\rightarrow} H \rightarrow 1,
$$

with $H$ a real algebraic group which is isomorphic to $\left(S^{1}\right)^{2 N-m}$ as a real Lie group. By taking a fiberwise $\left(S^{1}\right)^{m}$-compactification of $\left(\mathbb{R}^{*}\right)^{m}$, we obtain a compactification $\bar{G}_{\mathbb{R}}^{\prime}$ of the real algebraic variety $G_{\mathbb{R}}$.

Let us now give a more precise construction of $\bar{G}_{\mathbb{R}}^{\prime}$. Recall that $G$ is the extension of an abelian variety $A$ by a complex affine torus $T \cong\left(\mathbb{C}^{*}\right)^{m}$. For the moment, we stay in the category of complex algebraic varieties. As in Lemma 5.1, we can consider $G$ as a fiber product

$$
G \cong G_{1} \times_{A} G_{2} \times_{A} \cdots \times_{A} G_{m}
$$

where each $G_{i}$ is a $\mathbb{C}^{*}$-bundle over $A$. The associated line bundle of $G_{i}$ was denoted by $L_{i}$. Let $\bar{G}_{i}$ be the fiberwise projective compactification of $L_{i}$, i.e., the associated projective bundle of $L_{i}+\mathbb{C}_{A}$. Clearly, the $\mathbb{C P}^{1}$-bundle $\bar{G}_{i} \rightarrow A$ has two distinguished sections denoted by $0_{A}$ and $\infty_{A}$ corresponding to the fiberwise $0 \in \mathbb{C P}^{1}$ and $\infty \in \mathbb{C P}^{1}$, respectively. 
Now, we move to the category of real algebraic varieties. Denote the associated real algebraic variety of $\bar{G}_{i}$ by $\bar{G}_{i \mathbb{R}}$. The sections $0_{A}, \infty_{A} \subset \bar{G}_{i \mathbb{R}}$ are real subvarieties of codimension 2 . Define $\bar{G}_{i \mathbb{R}}^{\prime}$ to be the (real) blowup of $\bar{G}_{i \mathbb{R}}$ along both $0_{A}$ and $\infty_{A}$. Then, as a real manifold, $\bar{G}_{i \mathbb{R}}^{\prime}$ is a fiber bundle over $A_{\mathbb{R}}$, whose fibers are Klein bottles. The short exact sequence of complex algebraic groups

$$
1 \rightarrow \mathbb{C}^{*} \rightarrow G_{i} \rightarrow A \rightarrow 1
$$

induces a short exact sequence of real algebraic groups

$$
1 \rightarrow \mathbb{R}^{*} \rightarrow G_{i \mathbb{R}} \rightarrow H_{i} \rightarrow 1,
$$

where $H_{i}$ is a real algebraic group which is isomorphic to $\left(S^{1}\right)^{2 N-2 m+1}$ as a real Lie group. Clearly, $\bar{G}_{i \mathbb{R}}^{\prime}$ is a compactification of $G_{i \mathbb{R}}$ as a real algebraic variety, which fiberwise is a $S^{1}$-compactification of $\mathbb{R}^{*}$. Now, define

$$
\bar{G}_{\mathbb{R}}^{\prime}:=\bar{G}_{1 \mathbb{R}}^{\prime} \times_{A_{\mathbb{R}}} \cdots \times_{A_{\mathbb{R}}} \bar{G}_{m \mathbb{R}}^{\prime} .
$$

Then $\bar{G}_{\mathbb{R}}^{\prime}$ is a compactification of $G_{\mathbb{R}}$ as a real algebraic variety, which fiberwise is a $\left(S^{1}\right)^{m}$ compactification of $\left(\mathbb{R}^{*}\right)^{m}$.

In general, the map $\Phi_{\eta, G}$ may not extend to a real analytic map $\bar{G}_{\mathbb{R}}^{\prime} \rightarrow S^{1}$, because the homomorphism $\tau: G_{\mathbb{R}} \rightarrow H$ in (12) does not induce an isomorphism on the fundamental groups. In fact, the induced map $\tau_{*}: \pi_{1}\left(G_{\mathbb{R}}\right) \rightarrow \pi_{1}(H)$ is injective, and its cokernel is a 2-torsion group.

Lemma 5.3. Under the assumptions of Theorem 4.5, the map $2 \Phi_{\eta, G}:=\int 2 \operatorname{Re}(\eta): G \rightarrow S^{1}$ extends to a real analytic map $\overline{2 \Phi_{\eta, G}}: \bar{G}_{\mathbb{R}}^{\prime} \rightarrow S^{1}$.

Proof. By Lemma 5.2, $\Phi_{\eta, G}=\int \operatorname{Re}(\eta): G \rightarrow S^{1}$ contracts the fibers $\left(\mathbb{R}_{>0}\right)^{m}$ and factors through the map $\sigma: G=G_{\mathbb{R}} \rightarrow\left(S^{1}\right)^{2 N-m}$ in (11). By definition, the map $\tau: G_{\mathbb{R}} \rightarrow H$ in (12) factors through $\sigma: G=G_{\mathbb{R}} \rightarrow\left(S^{1}\right)^{2 N-m}$, and $2 \sigma$ factors through $\tau$. Thus, the map $2 \Phi_{\eta, G}$ factors through $\tau$. Note that $\tau$ is a real algebraic map (although $\sigma$ is not). Since with respect to (12) the real algebraic variety $\bar{G}_{\mathbb{R}}^{\prime}$ is a fiberwise $\left(S^{1}\right)^{m}$-compactification of $\left(\mathbb{R}^{*}\right)^{m}$, the map $2 \Phi_{\eta, G}:=\int 2 \operatorname{Re}(\eta): G \rightarrow S^{1}$ extends to a real analytic map $\overline{2 \Phi_{\eta, G}}: \bar{G}_{\mathbb{R}}^{\prime} \rightarrow S^{1}$.

Now, since $\bar{G}_{\mathbb{R}}^{\prime}$ is a compactification of $G_{\mathbb{R}}$ as a real algebraic variety, the closure $\bar{X}_{\mathbb{R}}$ of $X_{\mathbb{R}}$ in $\bar{G}_{\mathbb{R}}^{\prime}$ is a compactification of $X_{\mathbb{R}}$ as a real algebraic variety. Hence the complement $\bar{X}_{\mathbb{R}} \backslash X_{\mathbb{R}}$ is a real Zariski closed set in $\bar{X}_{\mathbb{R}}$. Since $\overline{2 \Phi_{\eta, G}}: \bar{G}_{\mathbb{R}}^{\prime} \rightarrow S^{1}$ is a real analytic map, for any $b \in S^{1}$, the corresponding fiber $\overline{2 \Phi}_{\eta, G}^{-1}(b)$ is a closed real analytic subvariety of $\bar{G}_{\mathbb{R}}^{\prime}$. Thus, for any $a \in S^{1}$, $\Phi_{\eta}^{-1}(a)$ consists of some connected components of

$$
X \cap \overline{2 \Phi}_{\eta, G}^{-1}(2 a)=\left(\bar{X}_{\mathbb{R}} \cap \overline{2 \Phi}_{\eta, G}^{-1}(2 a)\right) \backslash\left(\bar{G}_{\mathbb{R}}^{\prime} \backslash G_{\mathbb{R}}\right),
$$

which is a real analytic constructible set of a compact real analytic manifold. By the stratified Morse theory of Goresky-MacPherson (see [16, Theorem 2.2.1 and Section 10.8]), the real analytic constructible set $X \cap \overline{2 \Phi}_{\eta, G}^{-1}(2 a)$ is homotopy equivalent to a finite CW-complex. Therefore, as it consists of some of the connected components of the real analytic constructible $X \cap \overline{2 \Phi}_{\eta, G}^{-1}(2 a)$, the subset $\Phi_{\eta}^{-1}(a)$ is also homotopy equivalent to a finite $\mathrm{CW}$-complex. This completes the proof of Theorem 4.5 . 
5.2. Proof of Proposition 4.6. We first make the following simple observation.

Lemma 5.4. The short exact sequence (10) as a complex of complex Lie groups induces a canonical splitting of $G_{\mathbb{R}}$ as a direct sum of two real Lie groups

$$
G_{\mathbb{R}} \cong \mathbb{R}^{m} \oplus\left(S^{1}\right)^{2 N-m} .
$$

Proof. As a real Lie group, $G_{\mathbb{R}} \cong \mathbb{R}^{2 N} / \Lambda$, where $\Lambda$ is a discrete subgroup of $\mathbb{R}^{2 N}$ of rank $2 N-m$. Let $\Lambda_{\mathbb{R}}$ be the subspace of $\mathbb{R}^{2 N}$ generated by $\Lambda$. Then $\Lambda_{\mathbb{R}} / \Lambda \cong\left(S^{1}\right)^{2 N-m}$ is a Lie subgroup of $G_{\mathbb{R}}$. Obviously, the restriction of $\sigma: G_{\mathbb{R}} \rightarrow\left(S^{1}\right)^{2 N-m}$ to $\Lambda_{\mathbb{R}} / \Lambda$ is an isomorphism. Thus, the inclusion map $\Lambda_{\mathbb{R}} / \Lambda \rightarrow G$ gives rise to a splitting of the short exact sequence (11). Notice that $\mathbb{R}$ is isomorphic to $\mathbb{R}_{>0}$ as real Lie groups via the exponential map. Thus, the splitting (13) is canonical.

Proof of Proposition 4.6. Let $H$ be the space of left invariant real 1-forms on $G_{\mathbb{R}}$. The splitting (13) induces complementary subspaces $H_{\mathbb{R}^{m}}$ and $H_{\left(S^{1}\right)^{2 N-m}}$ of $H$.

Since every left invariant 1 -form on $G_{\mathbb{R}}$ is closed, we have a linear map

$$
\zeta \mapsto[\zeta]: H \rightarrow H^{1}(G ; \mathbb{R}) .
$$

By (13), the kernel of (14) is equal to $H_{\mathbb{R}^{m}}$, and the restriction of (14) to $H_{\left(S^{1}\right)^{2 N-m}}$ is an isomorphism.

Notice that the complex structure of $G$ gives rise to an action on $H$, which we denote by $J$. Then the map

$$
\xi \mapsto \xi+J \xi: H \rightarrow \Gamma
$$

is an inverse of the map Re $: \Gamma \rightarrow H$. Furthermore, it follows from a polar coordinate calculation as in the proof of Lemma 5.2 that for $\eta \in \Gamma,\left[\eta_{T}\right]$ is real if and only if $\operatorname{Re}(\eta) \in H_{\left(S^{1}\right)^{2 N-m}}$. Therefore, the restriction of the maps

$$
\Gamma \stackrel{\mathrm{Re}}{\longrightarrow} H \stackrel{[-]}{\longrightarrow} H^{1}(G ; \mathbb{R})
$$

induces two isomorphisms of real vector spaces

$$
\left\{\eta \in \Gamma \mid\left[\eta_{T}\right] \in H^{1}(T, \mathbb{C}) \text { is real }\right\} \rightarrow H_{\left(S^{1}\right)^{2 N-m}} \rightarrow H^{1}(G ; \mathbb{R}),
$$

thus proving the claim.

\section{Palais-Smale COnditions}

In this section we prove Theorem 4.2 and Theorem 4.10 .

First, we construct the Zariski open subset $U$ in Theorem 4.2. Recall that $\Gamma$ is the space of left invariant holomorphic 1-forms on $G, \operatorname{dim} G=N$ and $\operatorname{dim} X=n$. Define the degenerating locus 1 in $X \times \Gamma$ by

$$
Z:=\{(x, \eta) \in X \times \Gamma \mid \eta \text { vanishes at } x\} .
$$

Since the degenerating condition is given by algebraic equations, the locus $Z$ is an algebraic set of $X \times \Gamma$. Since $X$ is smooth, the first projection $p_{1}: Z \rightarrow X$ is a vector bundle with fiber dimension $N-n$. Thus, $\operatorname{dim} Z=N$, and the second projection $p_{2}: Z \rightarrow \Gamma$ is generically finite (e.g., by using again Verdier's generic fibration theorem). Let $U \subset \Gamma$ be the Zariski open set where $p_{2}: Z \rightarrow \Gamma$ is finite and étale, or, equivalently, a finite covering map. Since being finite and étale is an open condition, $U \subset \Gamma$ is a non-empty Zariski open subset.

\footnotetext{
${ }^{1}$ The definition of the degenerating locus $Z$ is motivated by the likelihood correspondence in algebraic statistics (see [18]).
} 
One can easily check that the holomorphic 1 -form $\eta_{X}$ degenerates at a point $x$ if and only if $Z$ intersects $X \times\{\eta\}$ at $(x, \eta)$. Moreover, the 1 -form $\eta_{X}$ has a regular singularity at $x$ if and only if $Z$ intersects $X \times\{\eta\}$ transversally at $(x, \eta)$. Since the projection $p_{2}: Z \rightarrow \Gamma$ is étale over $U$, and since $\eta \in U$, it follows that $Z$ intersects $X \times\{\eta\}$ transversally. Thus, the 1-form $\eta_{X}$ has a regular singularity at every degeneration point. By the holomorphic Morse lemma, the real part $\operatorname{Re}\left(\eta_{X}\right)$ also has a regular singularity at every degeneration point. Thus, the function $\Phi_{\eta}=\int \operatorname{Re}\left(\eta_{X}\right)$ is a circle-valued Morse function.

The following lemma follows immediately from the fact that $p_{2}$ is finite covering map over $U$.

Lemma 6.1. For any $\eta \in U$, there exists a small open neighborhood $U_{\eta}$ (in the sense of analytic topology) of $\eta$ in $U$, such that $\bar{U}_{\eta} \subset U$ and $p_{2}^{-1}\left(\bar{U}_{\eta}\right) \subset X \times \bar{U}_{\eta}$. Here $\bar{U}_{\eta}$ denotes the closure of $U_{\eta}$ in $\Gamma$. In particular, $p_{2}^{-1}\left(\bar{U}_{\eta}\right) \cap\left(X \times \bar{U}_{\eta}\right)=p_{2}^{-1}\left(\bar{U}_{\eta}\right)$ is compact.

Let $\mathfrak{g}_{\mathbb{R}}$ be the real Lie algebra of the real Lie group $G_{\mathbb{R}}$. Denote the real dual vector space of $\mathfrak{g}_{\mathbb{R}}$ by $\mathfrak{g}_{\mathbb{R}}^{\vee}$. Let $h_{e}$ be a Hermitian metric on the vector space $\mathfrak{g}_{\mathbb{C}}^{\vee}=\mathfrak{g}_{\mathbb{R}}^{\vee} \otimes_{\mathbb{R}} \mathbb{C}$, and let $h$ be the left invariant Hermitian metric on $G$ determined by $h_{e}$. Denote the restriction of $h$ to $X$ by $h_{X}$, and denote the associated Riemannian metric of $h_{X}$ by $g_{X}$. Since $\Phi_{\eta}: X \rightarrow S^{1}$ is defined as $\int \operatorname{Re}\left(\eta_{X}\right)$, we have $d \Phi_{\eta}=\operatorname{Re}\left(\eta_{X}\right)$. We claim the following:

$$
\begin{aligned}
\left\|\eta_{X}\right\|_{h_{X}}^{2} & =\left\|\operatorname{Re}\left(\eta_{X}\right)\right\|_{g_{X}}^{2}+\left\|\operatorname{Im}\left(\eta_{X}\right)\right\|_{g_{X}}^{2} \\
& =2\left\|\operatorname{Re}\left(\eta_{X}\right)\right\|_{g_{X}}^{2} \\
& =2\left\|\nabla \Phi_{\eta}\right\|_{g_{X}}^{2} .
\end{aligned}
$$

In fact, the first equality holds since $\eta_{X}$ is a holomorphic 1-form. The third equality follows from the definition of $\Phi_{\eta}$. To check the second equality at a point $x \in X$, let $z_{i}=x_{i}+\sqrt{-1} y_{i}$ be a set of unitary coordinates of $X$ at $x$. Since $\eta_{X}$ is a holomorphic 1-form, by the Cauchy-Riemann equation we have:

and

$$
\left\langle\operatorname{Re}\left(\eta_{X}\right), \partial / \partial_{x_{i}}\right\rangle=\left\langle\operatorname{Im}\left(\eta_{X}\right), \partial / \partial_{y_{i}}\right\rangle
$$

$$
\left\langle\operatorname{Re}\left(\eta_{X}\right), \partial / \partial_{y_{i}}\right\rangle=-\left\langle\operatorname{Im}\left(\eta_{X}\right), \partial / \partial_{x_{i}}\right\rangle
$$

Thus, at the point $x \in X$,

$$
\begin{aligned}
\left\|\operatorname{Im}\left(\eta_{X}\right)\right\|_{g_{X}}^{2} & =\sum_{i}\left(\left\langle\operatorname{Im}\left(\eta_{X}\right), \partial / \partial_{x_{i}}\right\rangle^{2}+\left\langle\operatorname{Im}\left(\eta_{X}\right), \partial / \partial_{y_{i}}\right\rangle^{2}\right) \\
& =\sum_{i}\left(\left\langle\operatorname{Re}\left(\eta_{X}\right), \partial / \partial_{y_{i}}\right\rangle^{2}+\left\langle\operatorname{Re}\left(\eta_{X}\right), \partial / \partial_{x_{i}}\right\rangle^{2}\right) \\
& =\left\|\operatorname{Re}\left(\eta_{X}\right)\right\|_{g_{X}}^{2} .
\end{aligned}
$$

Since $x$ is an arbitrary point in $X$, equality (15) holds.

Proof of Theorem 4.2. Suppose $S$ is a subset of $X$ on which $\left\|\nabla \Phi_{\eta}\right\|_{g_{X}}^{2}$ is not bounded away from zero, and assume that the closure of $S$ does not contain any degeneration point of $\eta_{X}$. Since $\left\|\nabla \Phi_{\eta}\right\|_{g_{X}}^{2}=\frac{1}{2}\left\|\eta_{X}\right\|_{h_{X}}^{2}$, it follows that there exists a sequence of points $\left(x_{i}\right)_{i=1}^{\infty}$ in $S$ such that $\lim _{i \rightarrow \infty}\left\|\left(\eta_{X}\right)_{x_{i}}\right\|_{h_{X}}=0$ and $\left(x_{i}\right)_{i=1}^{\infty}$ does not have any limit point in $X$.

To finish the proof, we need the following lemma.

Lemma 6.2. Suppose $\left\|\left(\eta_{X}\right)_{x}\right\|_{h_{X}}=\epsilon$ for some point $x \in X$. Then there exists a unique $\theta \in \Gamma$ such that $\|\theta\|_{h_{\Gamma}}=\epsilon$ and $\eta_{X}-\theta_{X}$ degenerates at $x$. Here, the norm $\|\cdot\|_{h_{\Gamma}}$ on $\Gamma$ is defined to be 
the restriction of the norm $\|\cdot\|_{h_{e}}$ on $\mathfrak{g}_{\mathbb{C}}^{\vee}$ via the natural isomorphisms $\Gamma \cong \mathfrak{g}^{\vee} \cong \mathfrak{g}_{\mathbb{C}}^{\vee(1,0)}$, where $\mathfrak{g}$ is the Lie algebra of the complex Lie group $G$, and $\mathfrak{g}^{\vee}$ is the dual of $\mathfrak{g}$ as a complex vector space.

Proof. The statement is local at $x$ and only involves the first order differential. Without loss of generality, we can assume that $G=\mathbb{C}^{N}$ with coordinates $\left(z_{j}\right)_{1 \leq j \leq N}$ and the standard Hermitian metric $h, X=\left\{z_{n+1}=\cdots=z_{N}=0\right\}$, and $x=(0, \ldots, 0)$. Since $\left\|\left(\eta_{X}\right)_{x}\right\|_{h_{X}}=\epsilon$, by a unitary coordinate change within $\left(z_{j}\right)_{1 \leq j \leq n}$, we can assume that

$$
\eta_{X}=\eta_{1} d z_{1}+\cdots+\eta_{n} d z_{n}
$$

where $\eta_{j}$ are local holomorphic functions on $X$ near $x$ with $\eta_{1}(x)=\epsilon$, and $\eta_{j}(x)=0$ for $2 \leq j \leq n$. Since $\theta$ is an left invariant holomorphic 1 -form on $G$, it is of the form

$$
\theta=\theta_{1} d z_{1}+\cdots+\theta_{N} d z_{N},
$$

where $\theta_{j} \in \mathbb{C}$ are constant. The condition that $\eta_{X}-\theta_{X}$ degenerates at $x$ is equivalent to $\theta_{1}=\epsilon$ and $\theta_{j}=0$ for $2 \leq j \leq n$. Now, the condition $\|\theta\|_{h_{\Gamma}}=\epsilon$ implies that $\theta_{j}=0$ for all $j>n$. Thus, $\theta=\epsilon d z_{1}$ is the unique left invariant holomorphic 1 -form satisfying the conditions in the lemma.

By Lemma 6.2, for each $i$ we can find $\theta_{i} \in \Gamma$ such that $\left\|\theta_{i}\right\|_{h_{\Gamma}}=\left\|\left(\eta_{X}\right)_{x_{i}}\right\|_{h_{X}}$ and $\eta_{X}-\left(\theta_{i}\right)_{X}$ degenerates at $x_{i}$. Thus, we have a sequence of points $\left(x_{i}, \eta-\theta_{i}\right)$ in $Z$. Since $\lim _{i \rightarrow \infty}\left\|\left(\eta_{X}\right)_{x_{i}}\right\|_{h_{X}}=$ 0 , we have $\lim _{i \rightarrow \infty}\left\|\theta_{i}\right\|_{h_{\Gamma}}=0$, or equivalently, $\eta-\theta_{i}$ converges to $\eta$ in $\Gamma$. Since $x_{i}$ does not have a limit point in $X$, the sequence $\left(x_{i}, \eta-\theta_{i}\right)$ does not have a limit point in $X \times \Gamma$. However, by Lemma 6.1] there exists an open neighborhood $U_{\eta}$ of $\eta$ in $\Gamma$ such that $p_{2}^{-1}\left(\bar{U}_{\eta}\right) \cap\left(X \times \bar{U}_{\eta}\right)$ is compact. Since $\eta-\theta_{i}$ converges to $\eta$, and since $\eta \in U$, when $i$ is sufficiently large we have $\eta-\theta_{i} \in U$. Hence, by Lemma [6.1] when $i$ is sufficiently large, $\left(x_{i}, \eta-\theta_{i}\right)$ is contained in the compact subset $p_{2}^{-1}\left(\bar{U}_{\eta}\right) \cap\left(X \times \bar{U}_{\eta}\right)$ of $Z$. This is a contradiction to the fact that $x_{i}$ does not have a limit point in $X$.

Thus, we have concluded the proof of Theorem 4.2.

The proof of Theorem 4.10 is very similar to that of Theorem 4.2 and it will be sketched below.

Proof of Theorem 4.10. First, we should replace $\mathbb{C}^{*}$ by $\mathbb{C} / \mathbb{Z}$, and write $\Psi_{\eta}=\int \eta_{X}: X \rightarrow \mathbb{C} / \mathbb{Z}$ in the theorem to be more precise. The map $\int \eta_{X}: X \rightarrow \mathbb{C} / \mathbb{Z}$ splits into the real part $\int \operatorname{Re}\left(\eta_{X}\right)$ : $X \rightarrow S^{1}=\mathbb{R} / \mathbb{Z}$ and the imaginary part $\int \operatorname{Im}\left(\eta_{X}\right): X \rightarrow \mathbb{R}$. Composing with the isomorphism $\mathbb{C} / \mathbb{Z} \cong \mathbb{C}^{*}$ defined by $z \mapsto e^{2 \pi \sqrt{-1} z}$, the map $|\cdot|: \mathbb{C}^{*} \rightarrow \mathbb{R}_{>0}$ becomes $z \mapsto e^{2 \pi \operatorname{Im}(z)}: \mathbb{C} / \mathbb{Z} \rightarrow \mathbb{R}_{>0}$. Hence,

$$
\Psi_{\eta}(x)=\log \left|\int_{e}^{x} \eta_{X}\right|=\log \left(e^{2 \pi \operatorname{Im} \int_{e}^{x} \eta_{X}}\right)=2 \pi \int_{e}^{x} \operatorname{Im}\left(\eta_{X}\right)
$$

where $\int_{e}^{x} \eta_{X} \in \mathbb{C}^{*}$ and $\int_{e}^{x} \operatorname{Im}\left(\eta_{X}\right) \in \mathbb{R}$. In other words, $\Psi_{\eta}=2 \pi \int \operatorname{Im}\left(\eta_{X}\right)$. Since $\eta_{X}$ is a holomorphic 1-form, by equation (15) we get:

$$
\left\|\nabla \Psi_{\eta}\right\|=2 \pi\left\|\operatorname{Im}\left(\eta_{X}\right)\right\|=\sqrt{2} \pi\left\|\eta_{X}\right\| .
$$

Suppose $S$ is a subset of $X$ on which $\left\|\nabla \Psi_{\eta}\right\|_{g_{X}}^{2}$ is not bounded away from zero, and suppose that the closure of $S$ does not contain any degeneration point of $\eta_{X}$. Then we can find a sequence $\left(x_{i}\right)_{i=1}^{\infty}$ in $S$ such that $\lim _{i \rightarrow \infty}\left\|\left(\eta_{X}\right)_{x_{i}}\right\|_{h_{X}}=0$ and $\left(x_{i}\right)_{i=1}^{\infty}$ does not have any limit point in $X$. Now, we are in the exact same situation as in the proof of Theorem 4.2, hence we can use the same argument to conclude the proof. 
Remark 6.3. In the proof of Theorem 4.10, we did not require $\left\|\Psi_{\eta}\right\|$ to be bounded on $S$. In other words, the function $\Psi_{\eta}: X \rightarrow \mathbb{R}$ satisfies the following stronger condition: if $S$ is a subset of $X$ on which $\left\|\nabla \Psi_{\eta}\right\|$ is not bounded away from zero, then there exists a critical point of $\Psi_{\eta}$ in the closure of $S$.

\section{REFERENCES}

[1] B. Bhatt, C. Schnell, P. Scholze, Vanishing theorems for perverse sheaves on abelian varieties, revisited, Selecta Math. (N.S.) 24 (2018), no. 1, 63-84.

[2] N. Budur, B. Wang, The signed Euler characteristic of very affine varieties, Int. Math. Res. Not. IMRN 2015, no. $14,5710-5714$.

[3] N. Budur, Y. Liu, B. Wang, The monodromy theorem for compact Kähler manifolds and smooth quasi-projective varieties, arXiv:1609.06478, Math. Ann. (published online), doi:10.1007/s00208-017-1541-3.

[4] C. De Concini, C. Procesi, On the geometry of toric arrangements, Transform. Groups 10 (2005), no. 3-4, 387-422.

[5] C. De Concini, C. Procesi, Topics in hyperplane arrangements, polytopes and box-splines, Universitext, Springer, New York, 2011.

[6] M. W. Davis, Complements of hyperplane arrangements as posets of spaces, arXiv:1502.03650 withdrawn.

[7] M. W. Davis, T. Januszkiewicz, I. J. Leary, The $L^{2}$-cohomology of hyperplane complements, Groups Geom. Dyn. 1 (3) (2007), 301-309.

[8] M. W. Davis, S. Settepanella, Vanishing results for the cohomology of complex toric hyperplane complements, Publ. Mat. 57 (2013), no. 2, 379-392.

[9] A. Dimca, Hyperplane arrangements, M-tame polynomials and twisted cohomology, Commutative Algebra, Singularities and Computer Algebra (Sinaia, 2002), 113-126, NATO Sci. Ser. II Math. Phys. Chem. 115, Kluwer Acad. Publ., Dordrecht, 2003.

[10] A. Dimca, A. Libgober, Regular functions transversal at infinity, Tohoku Math. J. (2) 58 (2006), no. 4, 549-564.

[11] A. Dimca, A. Némethi, Hypersurface complements, Alexander modules and monodromy, Real and complex singularities, 19-43, Contemp. Math., 354, Amer. Math. Soc., Providence, RI, 2004.

[12] M. Farber, Topology of closed one-forms, Mathematical Surveys and Monographs, 108. American Mathematical Society, Providence, RI, 2004.

[13] J. Franecki, M. Kapranov, The Gauss map and a noncompact Riemann-Roch formula for constructible sheaves on semiabelian varieties, Duke Math. J. 104 (2000), no. 1, 171-180.

[14] S. Friedl, L. Maxim, Twisted Novikov homology of complex hypersurface complements, Math. Nachr. 290 (2017), no. 4, 604-612.

[15] O. Gabber, F. Loeser, Faisceaux pervers l-adiques sur un tore, Duke Mathematical Journal 83 (1996), no. 3, pp. 501-606.

[16] M. Goresky, R. MacPherson, Stratified Morse theory, Ergebnisse der Mathematik und ihrer Grenzgebiete (3), 14. Springer-Verlag, Berlin, 1988.

[17] J. Huh, The maximum likelihood degree of a very affine variety, Compos. Math. 149 (2013), no. 8, $1245-1266$.

[18] J. Huh, B. Sturmfels, Likelihood geometry, Combinatorial algebraic geometry, 63-117, Lecture Notes in Math., 2108, Fond. CIME/CIME Found. Subser., Springer, Cham, 2014.

[19] T. Kohno, A. Pajitnov, Circle-valued Morse theory for complex hyperplane arrangements, Forum Math. 27 (2015), no. 4, 2113-2128.

[20] F. Loeser, C. Sabbah, Caractérisation des D-modules hypergéometriques irreductibles sur le tore, Comptes Rendus Serie I. 312 (1991), no. 10, 735 - 738, continued in 315 (1992), no. 12, 1263 -1264.

[21] W. Lück, $L^{2}$-invariants: Theory and Applications to Geometry and K-Theory, Ergebnisse der Mathematik und ihrer Grenzgebiete. 3. Folge. A Series of Modern Surveys in Mathematics, 44. Springer-Verlag, Berlin, 2002.

[22] L. Maxim, Intersection homology and Alexander modules of hypersurface complements, Comm. Math. Helv. 81 (2006), 123-155.

[23] L. Maxim, $L^{2}$-Betti numbers of hypersurface complements, Int. Math. Res. Not. Vol. 2014, No. 17, pp. 4665-4678.

[24] J. Milnor, Infinite cyclic coverings, 1968 Conference on the Topology of Manifolds (Michigan State Univ., E. Lansing, Mich., 1967) pp. 115-133 Prindle, Weber \& Schmidt, Boston, Mass. 
[25] J. Noguchi, J. Winkelmann, Nevanlinna theory in several complex variables and Diophantine approximation, Grundlehren der Mathematischen Wissenschaften. Springer, Tokyo, 2014.

[26] R.S. Palais, S. Smale, A generalized Morse theory, Bull. Amer. Math. Soc. 70 (1964), 165-172.

[27] C. Schnell, Holonomic D-modules on abelian varieties, Publ. Math. Inst. Hautes Études Sci. 121 (2015), 1-55.

[28] J.-L. Verdier, Stratifications de Whitney et théorème de Bertini-Sard, Inventiones Math. 36 (1976), $295-312$.

[29] C. Voisin, Hodge theory and complex algebraic geometry. II. Cambridge Studies in Advanced Mathematics, 77. Cambridge University Press, Cambridge, 2003

Department of Mathematics, Ku Leuven, Celestijnenlaan 200B, B-3001 Leuven, Belgium E-mail address: liuyq1117@gmail.com

Department of Mathematics, University of Wisconsin-Madison, 480 Lincoln Drive, Madison WI 53706-1388, USA.

E-mail address: maxim@math.wisc.edu

Department of Mathematics, University of Wisconsin-Madison, 480 Lincoln Drive, Madison WI 53706-1388, USA.

E-mail address: bwang274@wisc.edu 